\title{
An engineering assessment methodology for non-sharp defects in steel structures - Part I: Procedure development
}

\author{
A.J. Horn ${ }^{\mathrm{a}, *}$, A.H. Sherry ${ }^{\mathrm{b}}$ \\ ${ }^{a}$ Tata Steel Research Development and Technology, Swinden Technology Centre, Structural Integrity, Moorgate, Rotherham S60 3AR, UK \\ ${ }^{\mathrm{b}}$ Dalton Nuclear Institute, University of Manchester, Sackville Street, Manchester M60 1QD, UK
}

\section{A R T I C L E I N F O}

Article history:

Received 24 February 2011

Received in revised form

27 September 2011

Accepted 11 October 2011

\section{Keywords:}

Fracture toughness

Cleavage fracture

Crack

Notch

\begin{abstract}
A B S T R A C T
This Part I paper describes a new engineering assessment methodology for ferritic steel structures containing non-sharp defects within the context of a Failure Assessment Diagram (FAD) approach. Although the modification of the FAD for non-sharp defects can be applied whether the initiating failure mechanism is cleavage or ductile tearing, this paper focuses on cleavage fracture. The parameters describing the sensitivity of the material toughness to the notch effect can either be measured by testing notched specimens of the same thickness as the structure, or for cleavage fracture they can be obtained using look-up tables generated using the Weibull stress toughness scaling model. The other parameters in the procedure can either be conservatively estimated using simple equations or they can be determined more accurately using finite element analysis. Validation of the new method is presented in the companion Part II paper: this shows that assessments of U-notched $\mathrm{SE}(\mathrm{B})$ specimens have significantly reduced conservatism when using the new assessment methodology compared to the standard FAD approach for sharp cracks.
\end{abstract}

(c) 2011 Elsevier Ltd. All rights reserved.

\section{Introduction}

The structural integrity of engineering structures is conventionally assessed using defect assessment procedures based on fracture mechanics approaches [1,2]. For a real or postulated defect, the crack driving force under the loading conditions and temperature of interest is compared with the material fracture toughness. Such procedures assume flaws to be infinitely sharp: while this assumption may be appropriate for fatigue cracks, in other cases such as elongated voids or mechanical dents it can be an overconservative assumption that can lead to a pessimistic assessment of structures and significant under-estimation of their safety margin against fracture.

\subsection{Failure assessment diagram}

In the R6 guidance document [1] and the BS7910 standard [2], structural integrity assessments are carried out in the context of a Failure Assessment Diagram (FAD) approach in which the ordinate $K_{r}$ indicates the proximity to fracture. $K_{r}$ is defined by the ratio

\footnotetext{
* Corresponding author.

E-mail address: Anthony.J.Horn@tatasteel.com (A.J. Horn).
}

of the linear elastic stress intensity factor $K_{I}$ to the material toughness $K_{m a t}$. The abscissa $L_{r}$ indicates the proximity to failure by plastic collapse and is defined by the applied load $P$ normalised by the plastic limit load $P_{L} . K_{r}$ and $L_{r}$ are both proportional to $P$ and a linear loading line can be plotted on the FAD. Failure is predicted at its intersection with the failure assessment curve, represented by the equation:

$K_{r}=f\left(L_{r}\right)$ for $L_{r}<L_{r}^{\max }$

Where $f\left(L_{r}\right)$ represents the failure assessment curve, and $L_{r}^{\max }$ is the ratio of the uniaxial flow stress to the uniaxial yield stress $\sigma_{\mathrm{y}}$ defined at $0.2 \%$ plastic strain, where flow stress is defined as the mean of the ultimate tensile stress (UTS) and $\sigma_{\mathrm{y}}$.

FAD approaches are generally based on values of $K_{\text {mat }}$ derived from fatigue pre-cracked fracture toughness specimens tested according to well-defined standards, e.g [3-5]. Fracture mechanics specimens containing blunt notches instead of cracks exhibit higher values of effective toughness compared to sharp cracks [6-15]. However, as yet there are no standards that provide normative guidance on how to take benefit of the increased effective toughness associated with a non-sharp flaw in a structural integrity assessment. As such, the application of existing FAD methods to structures containing non-sharp defects is inherently conservative, and may be unduly so. 
Although several authors have modified the FAD for non-sharp defects [16-20], as yet there is no fully validated engineering approach for FAD modification available in structural integrity standards. To the authors' knowledge, the FITNET document [21] is the first procedure to contain informative guidance for treating non-sharp defects in the context of a FAD framework. However, the method requires detailed finite element analysis of the flawed structure making this approach unsuitable for routine engineering use. Furthermore, the approach is based on the linear elastic notch stress intensity factor (NSIF). Although this approach has been shown to adequately describe fracture from blunt notches for very brittle materials such as ceramics or glasses [7,22], it appears to be less suitable when significant plastic deformation occurs at the notch tip prior to fracture (e.g. in steels $[6,14,19])$. Conversely however, some experimental work has suggested that linear elastic approaches can successfully predict failure from notches even when significant plastic deformation precedes failure [23].

\subsection{J-integral and application to notches}

The primary use of the J-integral in fracture mechanics is to quantify the crack driving force. $J$ can be interpreted in several ways when applied to sharp cracks, for example the deformation energy and line integral definitions [24]. Experimentally, $J$ can be determined from pre-cracked fracture toughness specimens using relationships such as that in ESIS P2-92 [5]:

$J=\frac{\eta U}{B(W-a)}$

where $U=$ total area under the load vs. load-line displacement trace, $W$ is the specimen width, $a$ is the crack length and $\eta$ is a proportionality constant equal to 2.0 for single edge notch bend $\mathrm{SE}(\mathrm{B})$ specimens. Similar expressions, where $J$ is partitioned into elastic and plastic components, are provided in other testing standards $[3,25]$.

Several authors have used the $J$-integral for notch problems $[9,26-29]$ including a consideration of potential path dependency of the notch $J$-integral $[16,27]$. Chen and Lu [27] showed that for a Vshaped notch the traction-free surfaces contribute to the $J$-integral with the consequence that the $J$-integral becomes dependent on the contour used to define $J$. However, for a U-notch the parallel notch flanks do not contribute to $J$ and the $J$-integral remains path independent, as long as the contour completely encloses the notch root.

Critical notch- $J$ values have been measured experimentally, as described by a number of authors. Most studies are concerned with ductile tearing from notches in steels and experimental data is presented in the form of $J$ resistance curves [10-13]; fewer studies report single-point critical $J$ values for brittle notch behaviour [9].

In terms of $J$ definitions for notches, Roberti et al. [30] showed that the non-partitioned expression for measuring $J$ experimentally (Eq. (2)) was equal to the deformation energy definition for threepoint bend specimens with $0 \leq \rho / a_{0} \leq 0.05$. Furthermore, the equivalence of the deformation energy definition and the contour integral definition of $J$ for a notch was confirmed by Wang et al. [16] using finite element analysis.

\subsection{Objective and structure of paper}

This paper and its companion Part II paper [31] propose and validate a new engineering method for assessing structures that contain non-sharp defects within a FAD framework. The new assessment method is designed to be simple to apply from an engineering perspective: the parameters required in the new assessment method can either be estimated using simple equations and look-up tables, or they can be determined more accurately using elastic-plastic finite element analysis and/or material testing.

The paper is structured as follows. Section 2 describes the methodology used to develop the FAD for non-sharp defects. Section 3 presents the modified procedure together with look-up tables defining the material parameters required by the new procedure. Section 4 provides a discussion of the results and the main conclusions are summarised in Section 5.

The companion Part II paper [31] presents a series of worked examples. The Part II paper also considers the relationship between the new FAD modified for non-sharp defects and the constraintmodified FAD approach detailed in R6. A combined notch and constraint-modified FAD is presented and compared with a series of shallow, blunt notch SE(B) specimens.

\section{Methodology}

The methodology used to develop the notch-modified FAD approach comprises four parts. First, a series of finite element analyses of SE(B) specimens are described (Section 2.1). These are used to show that existing non-partitioned expressions for determining $J$ from pre-cracked $S E(B)$ specimens can also be used to estimate the $J$-integral from notched $\mathrm{SE}$ (B) specimens (Section 2.2). Secondly, R6 Option 3 failure assessment loci are derived for the notched $S E(B)$ specimens and found to be broadly independent of notch root radius (Section 2.3). Thirdly, a new methodology is proposed for modifying the FAD for notched structures (Section 2.4). This FAD modification can be applied irrespective of whether the initiating failure mechanism is cleavage or ductile tearing. Finally, a look-up table is derived for estimating the effective notch toughness $J_{\text {mat }}^{\rho}$, a required input for the proposed FAD-based methodology (Section 2.5). This look-up table is applicable only for cleavage fracture without prior ductile tearing. It has been outside the scope of the current work to develop a corresponding look-up table for ductile tearing initiation. Validation of the new methodology is described in the companion Part II paper [31].

\subsection{Finite element analysis of $S E(B)$ specimens}

Three-dimensional, large-strain, elastic-plastic, finite element (FE) analyses were used to simulate $\mathrm{SE}(\mathrm{B})$ specimens containing sharp cracks and blunt notches loaded in three-point bending. Five models with blunt notches were constructed, each corresponding to an $\mathrm{SE}(\mathrm{B})$ specimen with $a_{0} / W=0.5, B=W=12.5 \mathrm{~mm}$, and a different notch root radius $\rho$. The SE(B) model containing a blunt notch of $\rho / a_{0}=0.32$ is shown in Fig. 1 . Two fatigue pre-cracked

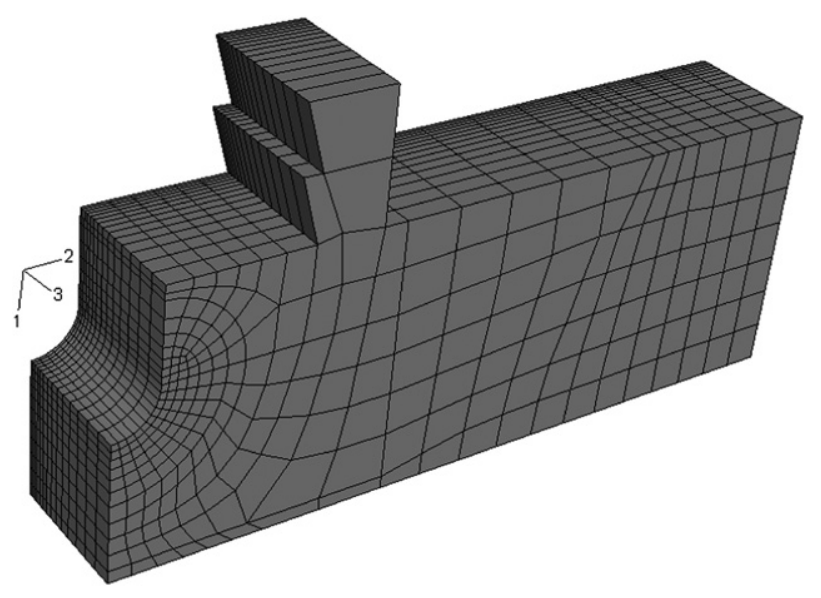

Fig. 1. One-quarter of $\operatorname{SE}(B)$ specimen with $\rho / a_{0}=0.32$. 
$\mathrm{SE}(\mathrm{B})$ specimens were modelled with $a_{0} / W$ ratios of 0.5 and 0.1 and a small notch root radius $\left(\rho / a_{0}=0.0004\right)$ to represent crack tip blunting. The value $\rho / a_{0}=0.0004$ was chosen because it is sufficiently small to ensure the initial radius has no effect on the Weibull stress at the loads required to initiate cleavage fracture (i.e. the Weibull stress vs. $J$ relationship becomes independent of the initial root radius when the deformed crack-tip radius is at least $\sim 3$ times the initial radius [32]).

Plasticity was modelled using a Ramberg Osgood fit to true-stress vs. true-strain curves obtained from tensile tests reported in reference [14]. Analyses were performed using ABAQUS version 6.6-1 [33].

Symmetry conditions were specified along the uncracked ligament $\left(x_{2}=0\right)$ and the longitudinal mid-plane $\left(x_{3}=6.25\right)$ thereby enabling one quarter of each $\mathrm{SE}(\mathrm{B})$ specimen to be modelled numerically. Each model consisted of quadratic 20-noded reduced integration hexahedral elements arranged into 14 variable thickness layers. The thickest element layer was defined at the longitudinal mid-plane with much thinner layers defined near the free surface to accommodate the reduced constraint in that region. Each model, including the pre-cracked SE(B), had a straight crack/notch front.

Within each of the 14 layers, rings of elements enclosed the notch tip as shown in Fig. 2. The notch tip elements had a dimension $l$ in the $\mathrm{x}_{1}$ direction and a dimension approximately equal to $l$ in the angular direction, $\theta=\tan ^{-1}\left(x_{2} / x_{1}\right)$. In the angular direction, 10 equally sized elements were defined in the range $0 \leq \theta \leq \pi / 2$ and $l$ was constant with $\theta$. In the $\mathrm{x}_{1}$ direction, $l$ increased with increasing distance $r$ from the centre of curvature of the notch tip, where $l=2 \pi r / 40$. The ratio $\rho / l$ at $r=\rho$ was therefore the same in all models, including the pre-cracked $S E(B)$ specimen modelled with a finite radius $\rho / a_{0}=0.0004$. This ensured consistency of mesh structure between models with notches of differing radii.

Three-point bending was simulated by applying a prescribed displacement in the 1-direction to a single node tied to the line of nodes defining the contact between the specimen and upper loading pin. This allowed for measurement of the reaction force through one node. The model was restrained by applying the boundary condition $u_{1}=0$ on a $1 \mathrm{~mm}$-wide region defining the contact between the specimen and lower supporting pin. This modelling technique is consistent with a similar FE model developed by Nevalainen and Dodds [34] which was used for validation purposes.

\subsection{J-integral for a notch}

The elastic-plastic finite element models described in Section 2.1 formed the basis for a comparison of the notch $J$-integral with several expressions for experimental determination of $J$ from precracked $S E(B)$ specimens. For this part of the study small-strain analyses were undertaken because the path dependence of the $J$ integral is less pronounced than for a finite-strain analysis [35]. The notch $J$-integral was evaluated on contours that completely enclosed the notch root. For each increment an averaged $J$ value was calculated by taking a weighted average of 15 individual $\mathrm{J}$ integral values evaluated along the notch front.

Four different expressions for experimental determination of $J$ were evaluated by post-processing each finite element model to obtain load, load-line displacement (LLD) and crack mouth opening displacement (CMOD). The expressions were taken from a range of testing standards for pre-cracked $\mathrm{SE}(\mathrm{B})$ specimens. It should be noted that the form of the equations presented here are for specimens with no side grooves.

ESIS P2-92 [5] provides a non-partitioned method for determining $J$ based on the total area $U^{L L D}$ under the load vs. LLD trace as follows:

$J=\frac{\eta^{L L D} U^{L L D}}{B(W-a)}$

where $\eta^{L L D}=2.0$.

Other standards differ from the ESIS procedure by requiring that $J$ is partitioned into elastic and plastic components. BS7448-1 [3] provides the following expression where the plastic component of $J$ is calculated from the plastic component of the area under the load vs. LLD trace $\left(U_{p}^{L L D}\right)$ as follows:

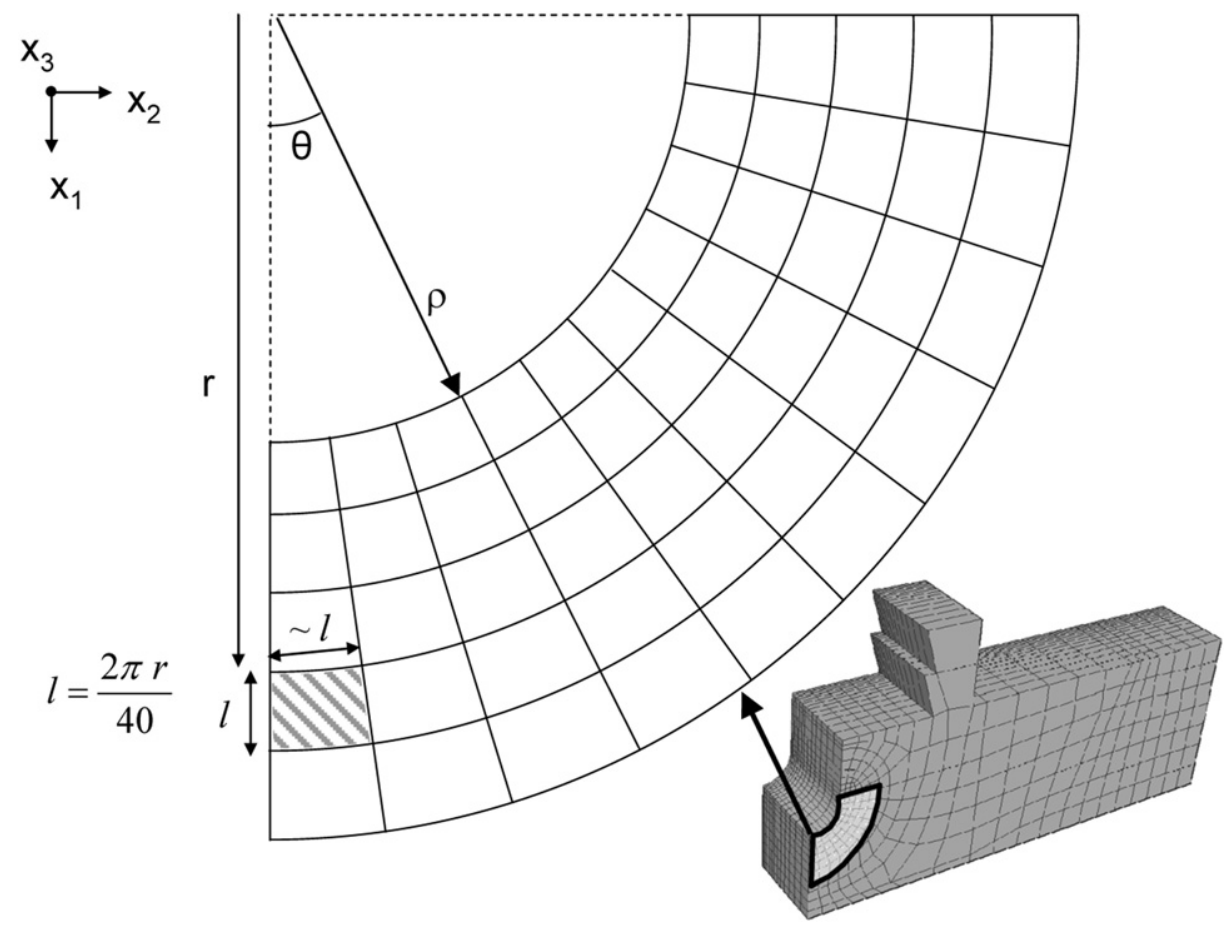

Fig. 2. Detail of the mesh ahead of the notch tip for all FE models. 
$J=\frac{K^{2}\left(1-v^{2}\right)}{E}+\frac{\eta_{p}^{L L D} U_{p}^{L L D}}{B\left(W-a_{0}\right)}$

where $\eta_{p}^{L L D}=2.0$.

The same equation is also found in ISO15653:2009 [36] and the (now superseded) ASTM E1820-08, except that $U_{p}^{L L D}$ is replaced by $U_{p}^{C M O D}$ which refers to the plastic component of the area under the load vs. CMOD trace. $\eta_{p}^{L L D}$ is replaced by $\eta_{p}^{C M O D}$ which is defined by:

$\eta_{p}^{C M O D}=3.785-3.101(a / W)+2.018(a / W)^{2}$

ASTM E1820-08a [25] contains a revised expression for $\eta_{p}^{C M O D}$ :

$\eta_{p}^{C M O D}=3.667-2.199(a / W)+0.437(a / W)^{2}$

A comparison of Eqs. (3)-(6) with the J-integral is shown in Fig. 3 for a pre-cracked $S E(B)$ specimen modelled with $\rho / a_{0}=0.0004$ and for a notched $\mathrm{SE}(\mathrm{B})$ specimen with $\rho / a_{0}=0.32$. For the pre-cracked $\mathrm{SE}(\mathrm{B})$ model, the experimental expressions are within $4 \%$ of the $J$-integral averaged along the crack front. For the notched $\mathrm{SE}(\mathrm{B})$ specimen with $\rho / a_{0}=0.32$, the experimental values of $J$ have a similar accuracy at high levels of applied $J$, but at low levels of loading the partitioned $J$ expressions underestimate the $J$ integral by up to $20 \%$. This can be attributed to the first term of Eq. (4) which uses the $K$ solution for a sharp crack. This term dominates when the plastic component of $J$ is small resulting in the observed errors in the calculation of $J$ for notched specimens. The ESIS method of calculating $J$ does not suffer from this limitation and provides the most accurate estimates of the $J$-integral (to within 5\%) at all levels of $J$ and $\rho$ studied.

These results are consistent with the conclusions of Roberti et al. [30] who showed the non-partitioned ESIS method agreed closely with the deformation energy definition of $J$. Wang et al. [16] also concluded that the deformation energy definition of $J$ is consistent with the energy-momentum-tensor definition. These are useful results as it means that experimental methods for measuring $J$
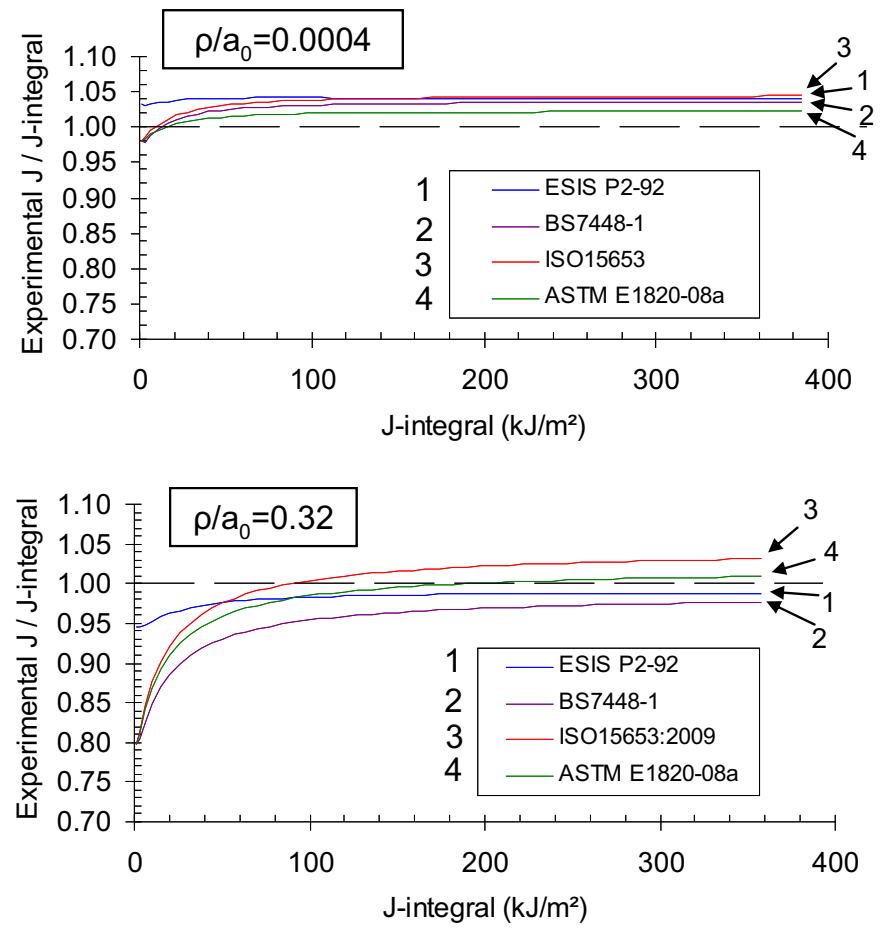

Fig. 3. Comparison of $J$-integral with experimental expressions for $J$ determination for pre-cracked and notched SE(B) specimens. originally developed for pre-cracked $\mathrm{SE}(\mathrm{B})$ specimens can also be used to estimate the $J$-integral for notched $\mathrm{SE}(\mathrm{B})$ specimens. However, care should be taken to avoid using partitioned estimates of $J$ for notched specimens especially at low levels of load. It is recommended that non-partitioned estimates of $J$ (such as the ESIS method) are used to estimate the J-integral for notched $\mathrm{SE}(\mathrm{B})$ specimens.

\subsection{Failure assessment curve}

R6 provides three main options for the definition of $f\left(L_{r}\right)$. The most accurate but most effort intensive to apply from an engineering perspective is the material and geometry-dependent Option 3 curve defined as:

$f_{3}\left(L_{r}\right)=\sqrt{\frac{J_{e}}{J}}$ for $L_{r}<L_{r}^{\max }$

where $J_{\mathrm{e}}$ and $J$ are the values of the $J$-integral determined from elastic and elastic-plastic finite element analyses respectively at a load corresponding to $L_{\mathrm{r}}$. For convenience R6 and BS7910 provide other Options which are easier to apply but at the expense of increased conservatism. The R6 Option 1 curve is material and geometry independent whereas the Option 2 curve is material dependent and requires knowledge of the material's stress-strain properties. It is important to note that none of these three failure assessment curve options have any relationship with the material fracture toughness.

Elastic and elastic-plastic FE models of the U-notched $\mathrm{SE}(\mathrm{B})$ specimens described in Section 2.1 were used to generate a family of Option 3 failure assessment curves for different notch root radii as follows:

$f_{3}\left(L_{r}^{\rho}\right)=\sqrt{\frac{J_{e}^{\rho}}{J^{\rho}}}$

where $J_{e}^{\rho}=$ notch $J$-integral determined from linear-elastic FE analysis

$J^{\rho}=$ notch $J$-integral determined using elastic-plastic FE analysis.

$L_{r}^{\rho}=$ load ratio defined using the notch limit load $\left(=P / P_{L}^{\rho}\right)$

For each $S E(B)$ specimen the notch limit load $P_{L}^{\rho}$ was determined from FE analysis using an elastic-perfectly plastic stress-strain relationship with yield stress equal to the engineering definition, i.e. the stress at $0.2 \%$ plastic strain.

The family of Option 3 curves is shown in Fig. 4 with the Option 1 and 2 curves shown for comparison. There is a weak dependence of the Option 3 notch failure assessment curves on $\rho / a_{0}$ : the safety region of the notch failure assessment curve reduces marginally

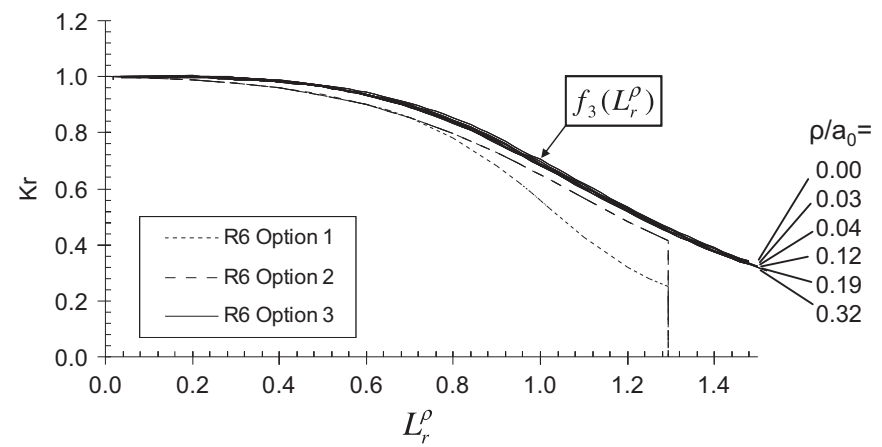

Fig. 4. R6 Option 3 failure assessment curves for $\mathrm{SE}(\mathrm{B})$ specimens of varying notch root radii. 
with increasing $\rho / a_{0}$, but the effect is small enough such that the Option 2 curve remains conservative (i.e. within the Option 3 curve) for all values of $\rho / a_{0}$ modelled. Wang et al. [16] also observed that Option 3 failure assessment curves are virtually independent of notch radius for four different U-notched plate geometries subjected to tension.

The broad independence of the Option 3 failure assessment curve with notch root radius does not contradict experimental observations of increasing resistance to fracture with increasing notch radius. The benefit associated with increasing notch radius is quantified by the increase in effective toughness; if this is used to modify the failure assessment point then there is no effect on the failure assessment curve.

The similarity of the notch and crack failure assessment curves is a very useful result from an engineering viewpoint: the existing R6 and BS7910 curves can be used directly without modification for the assessment of non-sharp defects. However, the coordinates of assessment points must be calculated in a manner consistent with that used to define the failure assessment curves, i.e.:

$L_{r}^{\rho}=P / P_{L}^{\rho}$

$K_{r}^{\rho}=\sqrt{\frac{J_{e}^{\rho}}{J_{\text {mat }}^{\rho}}}$

where $J_{\text {mat }}^{\rho}$ is an effective toughness associated with a notch of appropriate root radius. This is discussed in the following section.

\subsection{Proposed method for modifying the failure assessment point}

The benefit associated with a notch compared to a crack is quantified via the increase in effective toughness $J_{\text {mat }}^{\rho}$. This increase occurs whether the initiating failure mechanism is cleavage fracture or ductile tearing. In the context of the FAD, higher values of $J_{\text {mat }}^{\rho}$ produce lower values of $K_{r}^{\rho}$ moving the assessment point closer to the "safe" region defined within the failure assessment curve. The objective of this sub-section is to provide a method by which $J_{\text {mat }}^{\rho}$ can be determined for cleavage fracture, thereby allowing modification of the failure assessment point according to Eqs. (9) and (10). Although the increase in $J_{\text {mat }}^{\rho}$ occurs whether the failure mechanism is cleavage fracture or ductile tearing initiation, this sub-section is restricted to the mechanism of cleavage fracture.
The following empirical power law expression was found to describe the increase in effective cleavage toughness with increasing notch radius:

$\sqrt{\frac{J_{\text {mat }}^{\rho}}{J_{\text {mat }}}}=\frac{K_{\text {mat }}^{\rho}}{K_{\text {mat }}}=\left[1+\gamma\left(L_{r}^{\rho} / \beta_{N}\right)^{-l}\right]$

where $\gamma$ and $l$ are non-dimensional material properties that define the sensitivity of material toughness to the notch root radius. $\beta_{\mathrm{N}}$ is a non-dimensional and load-independent measure of the notch geometry defined such that $\beta_{\mathrm{N}}$ increases with increasing notch radius ( $\beta_{\mathrm{N}}=0$ for a crack). $\beta_{\mathrm{N}}$ defines the relationship between $L_{r}^{\rho}$ and the loading severity at the notch tip expressed in terms of an elastic notch tip stress $\sigma_{\mathrm{N}}$ :

$\frac{L_{r}^{\rho}}{\beta_{N}}=\frac{\sigma_{N}}{\sigma_{y}}$

$\sigma_{\mathrm{N}}$ is defined as the elastic stress acting at the tip of the notch in a direction perpendicular to the plane of the notch. $\sigma_{\mathrm{N}}$ scales with load: a given value of $\sigma_{\mathrm{N}}$ could correspond to an acute notch under low load or a blunter notch subject to a higher load.

Eq. (11) defines the failure locus shown in Fig. 5. A loading line may be plotted on the diagram for a notched component of interest with failure being predicted by its intersection with the failure locus. The curvature of the loading line is determined by $\beta_{\mathrm{N}}$ : blunt notches with high $\beta_{\mathrm{N}}$ values rise steeply and failure is predicted at large $J_{\text {mat }}^{\rho}$ values. As $\rho \rightarrow 0, \beta_{\mathrm{N}} \rightarrow 0$, the gradient of the loading line approaches the horizontal and $J_{\text {mat }}^{\rho} \rightarrow J_{\text {mat }}$.

\subsection{Derivation of look-up tables for material properties $\gamma$ and $l$}

The material properties $\gamma$ and $l$ in Eq. (11) define the notchsensitive toughness as a function of load (Fig. 5). For cleavage fracture these parameters may be derived using the Weibull stress based toughness scaling model which has previously been successfully applied to predict cleavage fracture from $S E(B)$ specimens with U-notches and cracks [14].

\subsubsection{Weibull cleavage fracture model}

The Beremin model [37] describes the proximity to cleavage fracture by use of the scalar Weibull stress, $\sigma_{\mathrm{w}}$. In its simplest form the probability of cleavage fracture is described by a two-parameter Weibull distribution:

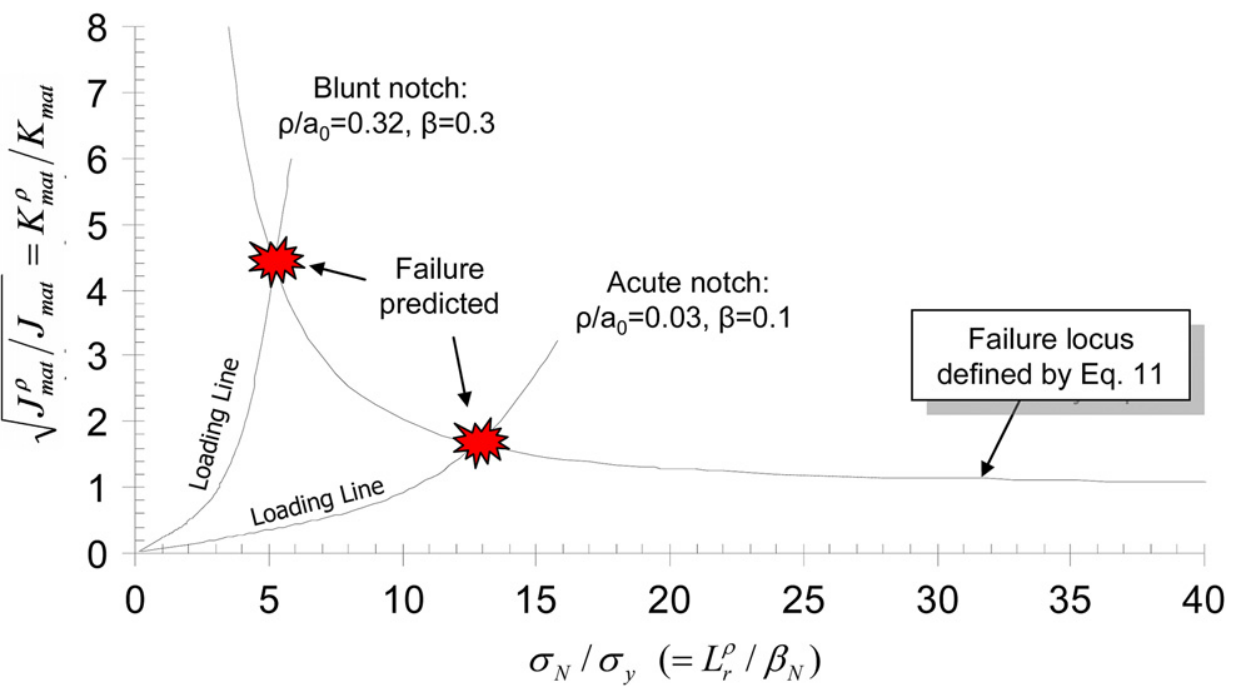

Fig. 5. Failure locus in toughness- $\sigma_{\mathrm{N}}$ space. 
$P_{f}=1-\exp \left[-\left(\frac{\sigma_{w}}{\sigma_{u}}\right)^{m}\right]$

where $\sigma_{\mathrm{u}}$ and $m$ are the Weibull parameters. The Weibull stress is calculated by integrating a weighted value of the maximum principal stress $\sigma_{1}$ over the plastic zone ahead of the stress concentration:

$\sigma_{w}=\left[\frac{1}{V_{0}} \int_{V} \sigma_{1}^{m} \mathrm{~d} V\right]^{1 / m}$

The constant $V_{0}$ is a reference volume required to ensure dimensional consistency and in the current work is taken as unity. The Weibull parameters $m$ and $\sigma_{\mathrm{u}}$ are determined by matching values calculated from the Beremin model to experimental values of cleavage fracture toughness. Reliable estimation of the Weibull parameters is only possible using experimental data that cover two different constraint levels. The method proposed by Gao et al. [38] provides a suitable methodology, as described in Section 3.3 of the companion Part II paper [31].

\subsubsection{Finite element analysis of small scale yielding}

A plane strain "boundary-layer" FE model was used to simulate a notch in an infinite body. Plane strain boundary conditions were applied to both faces in the 3-direction, so the model was essentially 2D despite the same 3D elements being used as for the $\mathrm{SE}(\mathrm{B})$ models, i.e. quadratic 20 -noded reduced integration elements. The model consisted of a semi-circular mesh of initial radius $R$ containing a radial notch modelled with a notch tip radius $\rho=2.5 \mu \mathrm{m}$. The ratio $R / \rho$ was set at $10^{5}$ to ensure that the notch tip plastic zone did not approach the boundary of the model thereby ensuring small-scale yielding conditions were preserved. The thickness of the model B was fixed at $12.5 \mathrm{~mm}$ and symmetry conditions were specified along the uncracked ligament $\left(x_{2}=0\right)$. All analyses were large-strain and performed using ABAQUS version 6.6-1 [33]. The increment size was fixed at 0.005 resulting in 200 increments.

Displacement boundary conditions were applied incrementally to the nodes on the outer edge of the model. These displacements were consistent with the leading, $K_{\mathrm{I}}$-dominated term of the Williams expansion [39] for the displacement field at a crack tip, as follows:

$u_{1}=K_{I} \frac{1+v}{E} \sqrt{\frac{R}{2 \pi}} \cos \left(\frac{\theta}{2}\right)(3-4 v-\cos \theta)$

$u_{2}=K_{I} \frac{1+v}{E} \sqrt{\frac{R}{2 \pi}} \sin \left(\frac{\theta}{2}\right)(3-4 v-\cos \theta)$

where the polar coordinates $R$ and $\theta$ define the position of the node with respect to the notch tip. Plasticity was modelled using tensile properties defined according to:

$\varepsilon / \varepsilon_{0}=\left(\sigma / \sigma_{0}\right)$ for $\varepsilon \leq \varepsilon_{0}$

$\varepsilon / \varepsilon_{0}=\left(\sigma / \sigma_{0}\right)^{n}$ for $\varepsilon>\varepsilon_{0}$

where $\varepsilon$ is the total true strain, $\sigma_{0}$ is the limit of proportionality being related to $\varepsilon_{0}$ through the relationship $\varepsilon_{0}=\sigma_{0} / E$, where $E$ is Young's modulus.

The range of tensile properties used in this work were chosen to match the range reported in reference [40] because this range bounds the flow properties for a wide range of ferritic steels $[41,42]$, including the material used to validate the method [31], as shown in Fig. $6\left(\sigma_{\mathrm{y}}\right.$ is the yield stress defined at $0.2 \%$ plastic strain). Boundary layer FE analyses were undertaken to sample a range of properties within the area illustrated in Fig. 6. Just under 200 combinations of $n$ and $E / \sigma_{\mathrm{y}}$ were modelled. The large number of models was necessary to ensure results were available with sufficient resolution to enable accurate interpolation between adjacent combinations of $n$ and $E / \sigma_{\mathrm{y}}$.

\subsubsection{Post-processing}

Numerical data (maximum principal stresses and integration point volumes) were post-processed from each boundary layer model to define the evolution of $\sigma_{\mathrm{w}}$ as a function of the notch $J$ integral $J^{\rho}$, for a given value of $m$. Values of $m$ were chosen to be consistent with those used in reference [40], i.e. from 5 to 20 in increments of 2.5. Three additional values of $m$ were chosen above 20 , also in increments of 2.5 , resulting in a total of ten $m$ values ranging from 5 to 27.5 .

Scaling functions were derived such that the $\sigma_{\mathrm{w}}$ vs. $J^{\rho}$ relationship obtained from one boundary layer analysis with notch root radius $\rho$ could be scaled to any other notch root radius $\rho^{\prime}$ while keeping the notch front length $B$ constant. Using dimensional arguments (see Appendix A) it can be shown that

$\frac{\left(J^{\rho}\right)^{\prime}}{J^{\rho}}=\frac{\rho^{\prime}}{\rho}$

$\frac{\sigma_{w}^{\prime}}{\sigma_{w}}=\left(\frac{\rho^{\prime}}{\rho}\right)^{2 / m}$

where prime superscripts denote scaled quantities. To verify Eqs. (19) and (20), a series of boundary layer models were analysed with different $\rho$ values but a constant $R / \rho$ ratio of $10^{5}$. Using Eqs. (19) and (20) the $\sigma_{\mathrm{w}}$ vs. $J^{\rho}$ relationship obtained from each boundary layer model was scaled to the same $\rho / B$ value of 0.080 . As shown in Fig. 7, the scaled $\sigma_{\mathrm{w}}$ vs. $J^{\rho}$ relationship is independent of the original value of $\rho$ prior to scaling, confirming that the scaling functions can be used as intended.

For each set of tensile data defined in Fig. 6, the following analysis steps were performed:

- Firstly, numerical data were post-processed to determine the $\sigma_{\mathrm{w}}$ vs. $J^{\rho}$ relationship for the single value of $\rho$ used in the boundary layer model.

- Secondly, scaled $\sigma_{\mathrm{w}}$ vs. $J^{\rho}$ relationships for 24 discrete values of $\rho^{\prime}$ were generated using Eqs. (19) and (20) These are shown as

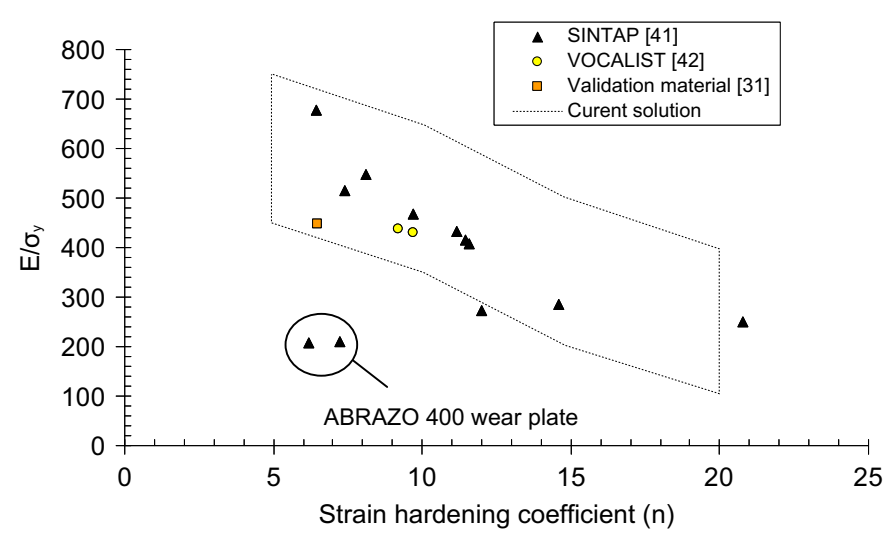

Fig. 6. Comparison of tensile properties used in the present work with those for a range of ferritic steels [40]. 


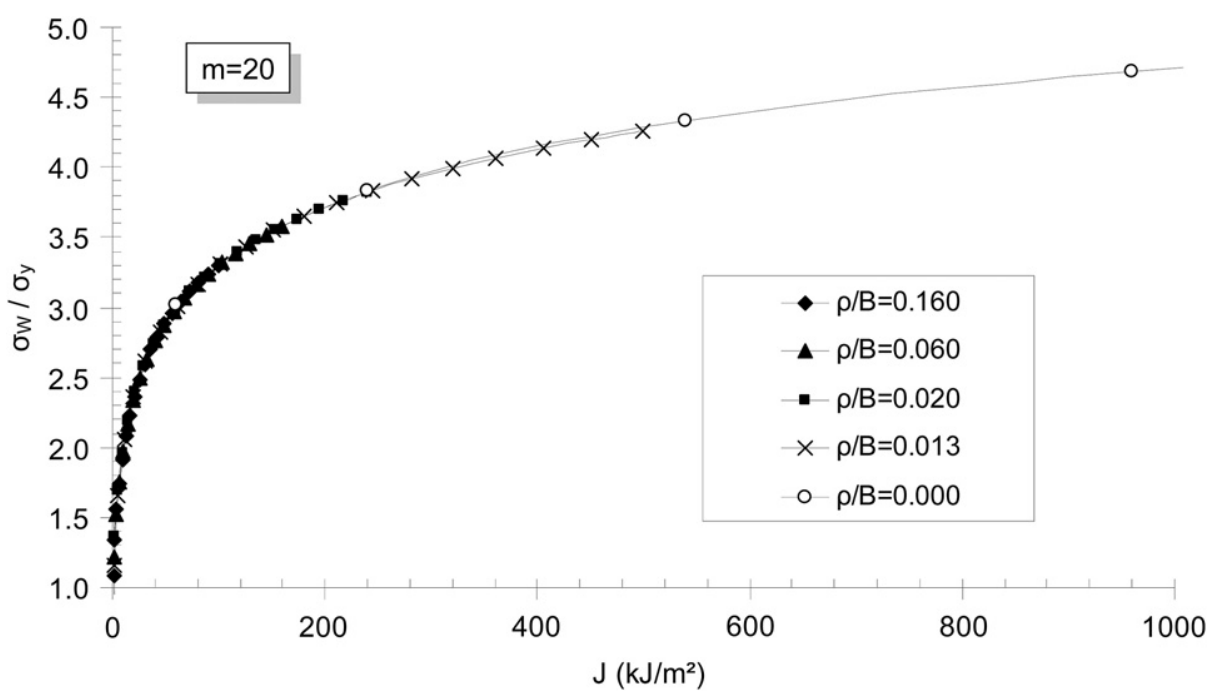

Fig. 7. $\sigma_{\mathrm{w}}-J$ relationship for $\rho / B=0.080$ derived by applying scaling function (Eqs. (19) and (20)) to boundary layer models of different notch tip radii ( $n=6.5$ ).

solid lines in Fig. 8 and correspond to loading lines for specific values of $\rho^{\prime}$.

- Thirdly, a locus of constant $\sigma_{\mathrm{N}} / \sigma_{\mathrm{y}}$ in $\sigma_{\mathrm{w}}-J^{\rho}$ space was derived by selecting the $24\left(\sigma_{\mathrm{w}}, J^{\rho}\right)$ datum pairs that have the same $\sigma_{\mathrm{N}} / \sigma_{\mathrm{y}}$ value. The dashed line in Fig. 8 is an example of such a locus.

- The previous step was repeated many times to generate a family of loci of constant $\sigma_{\mathrm{N}} / \sigma_{\mathrm{y}}$ as shown in Fig. 9

The probability of fracture is directly related to the Weibull stress via Eq. (13). Therefore, a horizontal line plotted in Fig. 9 defines a particular cleavage fracture probability. A perfectly sharp crack would be represented by the curve $\sigma_{\mathrm{N}} / \sigma_{\mathrm{y}}=\infty$, but in this case the curve $\sigma_{\mathrm{N}} / \sigma_{\mathrm{y}}=30$ is taken to approximate to a sharp crack. The implications of this approximation will be discussed later.

Using the horizontal line as a reference, the value $\left[J^{\rho}\right]_{30}$ approximately equates to $J_{m a t}$ for a given failure probability. The curves corresponding to lower values of $\sigma_{\mathrm{N}} / \sigma_{\mathrm{y}}$ define the variation of toughness with cleavage fracture probability for reduced notch tip loading severity. Using again the horizontal line as a reference, the values $\left[J^{\rho}\right]_{6}$ to $\left[J^{\rho}\right]_{4}$ define the notch-sensitive toughness at the same cleavage fracture probability, i.e. $J_{m a t}^{\rho}$. Therefore, the data presented in Fig. 9 enable to ratio of $J_{\text {mat }}^{\rho} / J_{\text {mat }}$ to be defined as a function of $\sigma_{\mathrm{N}} / \sigma_{\mathrm{y}}$ for a given probability of cleavage fracture.

A useful result is that for a given value of $\sigma_{\mathrm{N}} / \sigma_{\mathrm{y}}$, the ratio $J_{\text {mat }}^{\rho} / J_{\text {mat }}$ (and $K_{\text {mat }}^{\rho} / K_{\text {mat }}$ ) remains almost independent of $J^{\rho}, \sigma_{\mathrm{w}}$ and therefore $P_{\mathrm{f}}$ as shown in Fig. 10. It is this observation which enables a ratio of

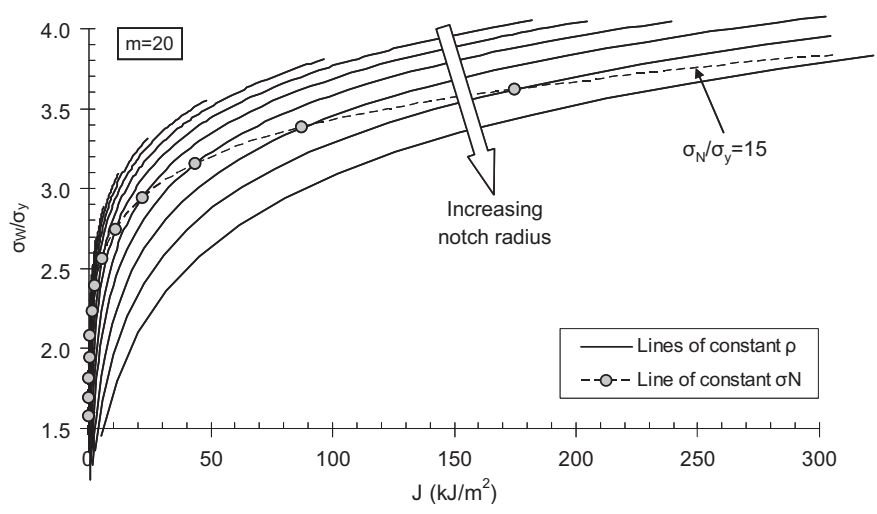

Fig. 8. Evolution of $\sigma_{\mathrm{W}}$ with $\mathrm{J}^{\rho}$ for constant $\rho$.
$K_{\text {mat }}^{\rho} / K_{\text {mat }}$ to be derived from these solutions which is independent of cleavage fracture probability. The resulting parameters $\gamma$ and $l$ that define this ratio as a function of $\sigma_{\mathrm{N}} / \sigma_{\mathrm{y}}$ (as shown in Fig. 5) are therefore also independent of cleavage fracture probability. The cleavage fracture probability is introduced when a value is assigned to $K_{\text {mat }}$. For example, a $K_{\text {mat }}$ value corresponding to a $5 \%$ cleavage fracture probability would enable $K_{\text {mat }}^{\rho}$ at that same cleavage fracture probability to be defined using the $\gamma$ and $l$ parameters with Eq. (11).

Values of $\gamma$ and $l$ were derived by curve fitting the $K_{\text {mat }}^{\rho} / K_{\text {mat }}$ vs. $\sigma_{\mathrm{N}} / \sigma_{\mathrm{y}}$ data as illustrated by Fig. 11. As stated earlier, the condition $\sigma_{\mathrm{N}} / \sigma_{\mathrm{y}}=30$ was taken to approximate to a sharp crack. This has two consequences. Firstly the $K_{\text {mat }}$ value used to normalise $K_{\text {mat }}^{\rho}$ will be marginally higher than the value for a truly sharp crack (a maximum difference of $10 \%$ at $\sigma_{\mathrm{N}} / \sigma_{\mathrm{y}}=30$ ); this reduces the magnitude of the ratio $K_{m a t}^{\rho} / K_{\text {mat }}$ and is therefore slightly conservative. Secondly, the ratio $K_{m a t}^{\rho} / K_{\text {mat }}$ calculated from the FE results equals unity when $\sigma_{\mathrm{N}} / \sigma_{\mathrm{y}}=30$ rather than $\sigma_{\mathrm{N}} / \sigma_{\mathrm{y}}=\infty$. Both anomalies are avoided in the final curve fit by only fitting to data points below $\sigma_{\mathrm{N}} / \sigma_{\mathrm{y}}=18$ and forcing the fitted curve to be asymptotic to $K_{\text {mat }}^{\rho} / K_{\text {mat }}=1$, as shown in Fig. 11.

Calculations were undertaken using the tensile properties assumed in Fig. 6 using $m$ values between 5 and 27.5. Values of $\gamma$ and $l$ were derived by curve fitting the $K_{\text {mat }}^{\rho} / K_{\text {mat }}$ vs. $\sigma_{N} / \sigma_{y}$ data for $\sigma_{N} / \sigma_{y}<18$. The resulting curves were found to be largely independent of $E / \sigma_{\mathrm{y}}$ (as shown in Fig. 12), being dependent only on $n$ and $m$ (Fig. 13 shows the dependence on $n$ for $m=20$ ). Values of $\gamma$ and $l$ for various combinations of $m$ and $n$ are tabulated in Section 3.2.

\subsection{Development of basic notch correction method}

Although $\sigma_{N}, J_{e}^{\rho}$ and $P_{\mathrm{L}}^{\rho}$ can be derived from a finite element analysis of the structure containing the non-sharp defect, from an engineering perspective it is desirable that simple expressions are also available for estimating these parameters.

A convenient expression for $\sigma_{N}$ was derived by Shin [43] based on the Creager-Paris elastic stress distribution ahead of a slender notch [44]:

$\sigma_{N}=\sigma\left(1+2 Y \sqrt{\frac{a}{\rho}}\right)$

where $\sigma$ is the remotely applied tensile stress and $Y$ is the geometry correction factor for a given geometrical configuration which is available in handbooks, e.g [45]. For a surface flaw $a$ is the flaw 


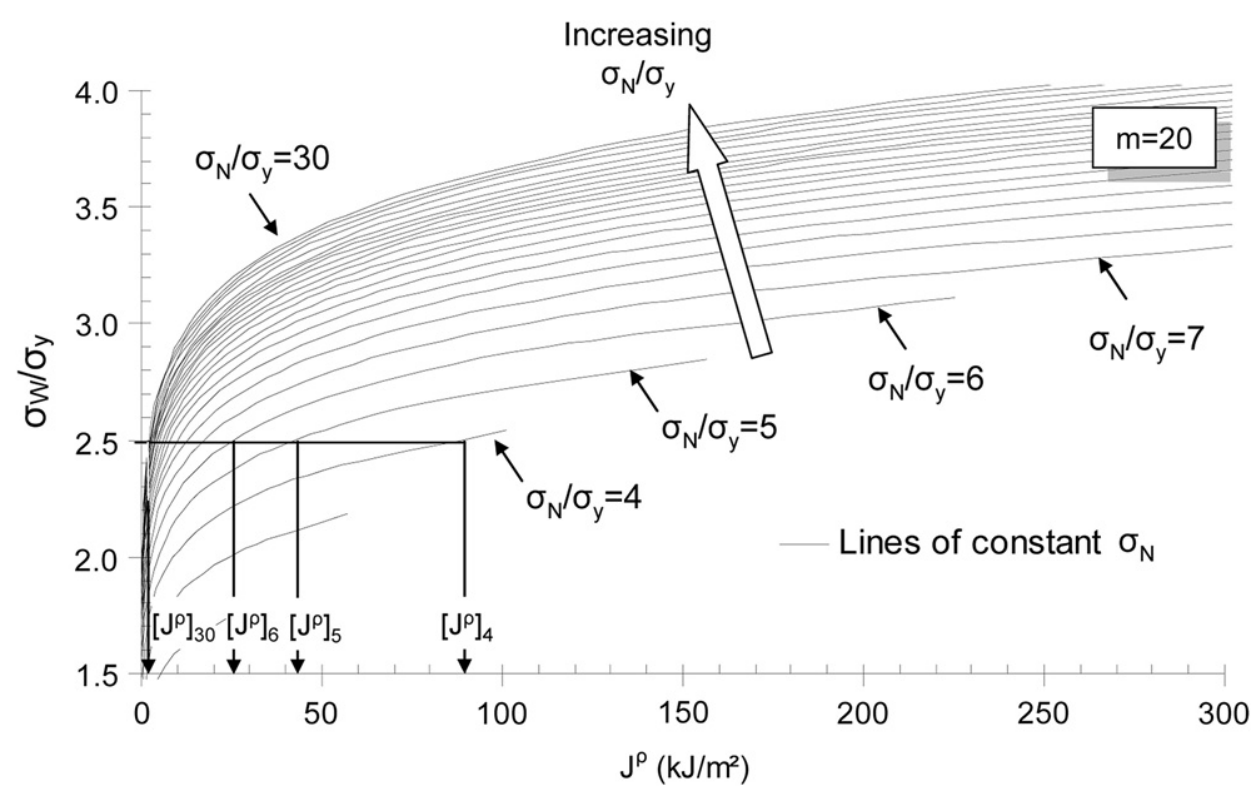

Fig. 9. Evolution of $\sigma_{\mathrm{W}}$ with $J^{\rho}$ for constant $\sigma_{\mathrm{N}} / \sigma_{\mathrm{y}}$.

depth, or for an internal flaw $a$ is the half-flaw length. For $\mathrm{SE}(\mathrm{B})$ specimens, replacing $\sigma$ with the expression for the elastic outer fibre stress results in the following expression:

$\sigma_{N}=\frac{3 P S}{2 B W^{2}}\left(1+2 Y \sqrt{\frac{a}{\rho}}\right)$

Eq. (22) was compared with linear elastic finite element models of $\mathrm{SE}$ (B) specimens and was found to be accurate to within $2.7 \%$ for the geometrical range evaluated $\left(0.0004 \leq \rho / a_{0} \leq 0.32\right)$.

Several authors have also derived convenient expressions for $\mathrm{Je}_{\mathrm{e}}^{\rho}$ by evaluating the $J$-integral along the arc defined by the notch tip [26,46-48]. Assuming no external tractions act on the notch surface the $J$-integral is given by

$J=\int_{-\pi / 2}^{+\pi / 2} W(\theta) \rho \cos (\theta) \mathrm{d} \theta$

where $\theta$ is the angular coordinate of points on the notch tip and
$W(\theta)$ is the strain energy density. As a first approximation, Matvienko [26] assumed the distribution of $W(\theta)$ to be

$W(\theta)=W_{\max } \cos \theta$

where $W_{\max }$ is the maximum strain energy density at the notch tip, which for linear elastic plane strain conditions is given by [46]:

$W_{\max }=\frac{\sigma_{N}^{2}\left(1-\nu^{2}\right)}{2 E}$

Substituting Eqs. (25) and (24) into (23) and integrating around the notch tip results in the following expression:

$J_{e}^{\rho}=\frac{\pi \rho \sigma_{N}^{2}\left(1-\nu^{2}\right)}{4 E}$

Eq. (24) is strictly valid only for $\rho / a_{0} \rightarrow 0$; consequently Eq. (26) is most accurate for acute notches and increasingly overestimates $J_{e}^{\rho}$ with increasing $\rho / a_{0}$. Comparison of Eq. (26) with linear elastic finite element analyses of the notched $\mathrm{SE}(\mathrm{B})$ specimens showed

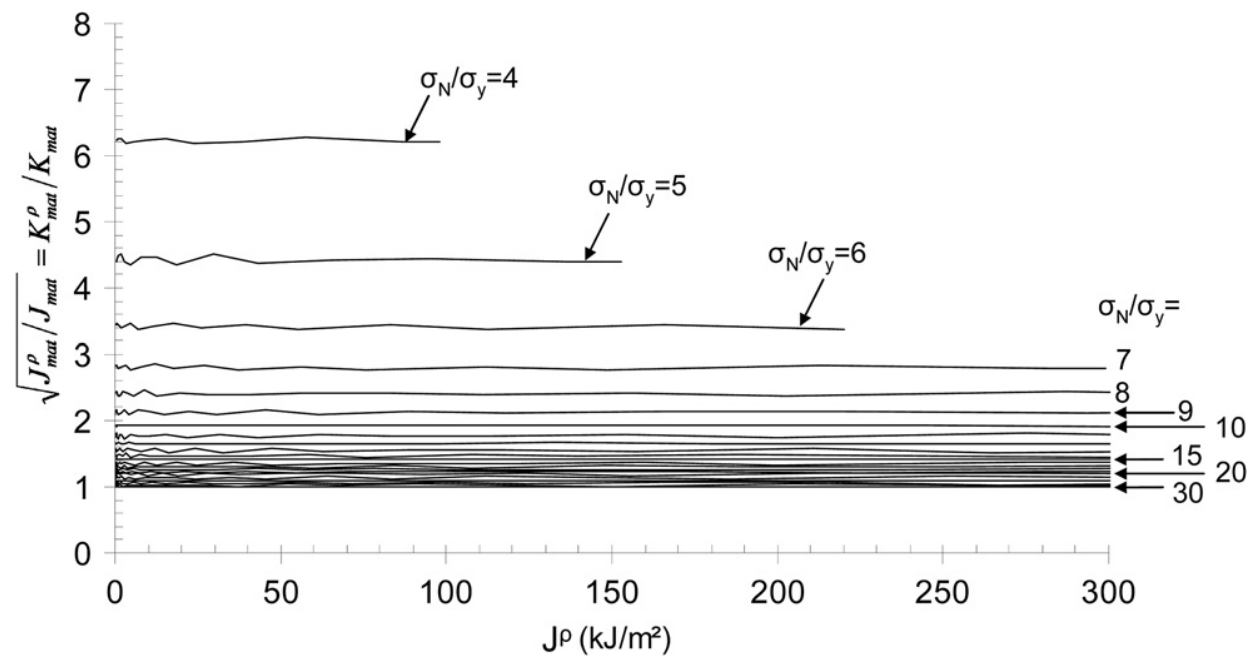

Fig. 10. Variation of $\mathrm{K}_{\mathrm{mat}}^{\rho} / \mathrm{K}_{\mathrm{mat}}$ with J for different values of $\sigma_{\mathrm{N}} / \sigma_{\mathrm{y}}$. 


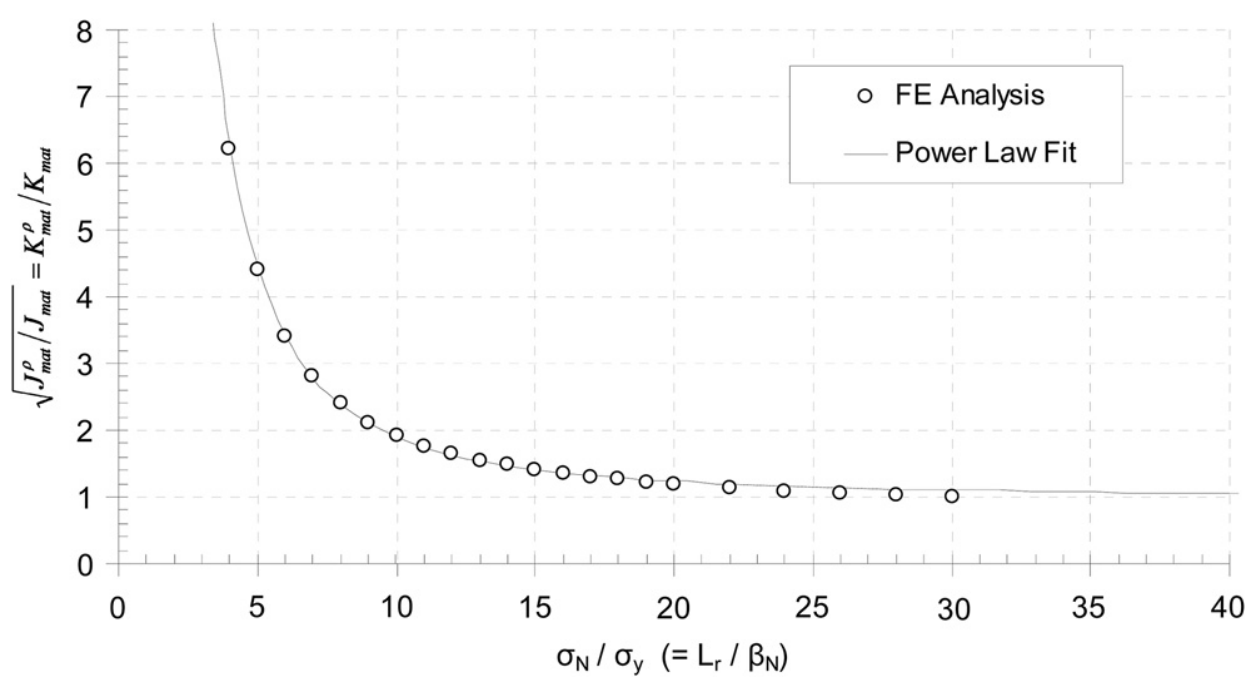

Fig. 11. Variation of $K_{m a t}^{\rho} / K_{m a t}$ with $\sigma_{\mathrm{N}} / \sigma_{\mathrm{y}}$ derived by FE and fitted with a power law expression for $\sigma_{\mathrm{N}} / \sigma_{\mathrm{y}}<18$.

accuracy of $J_{e}^{\rho}$ to be within $10 \%$ for $0.0004 \leq \rho / a_{0} \leq 0.04$ and within $15 \%$ for $0.04<\rho / a_{0} \leq 0.32$. Given that Eq. (26) tends to overestimate $J_{e}^{\rho}$ for notches with low acuity, the equation is therefore conservative for the purposes of the modified FAD approach.

It should be noted that more accurate forms of Eq. (24) have been proposed by Berto et al. [46] and Szanto and Read [47] as follows:

$W(\theta)=W_{\max } \cos ^{\delta} \theta$

where $\delta$ is a geometry-dependent function determined by finite element analysis. Although Berto et al. [46] derived some useful expressions for $\delta$ based on FE analysis these are valid only for $a_{0} / W \leq 0.2$ and are not applicable in the present case. For the engineering method proposed here, Eq. (26) can be used to provide conservative estimates of $J_{e}^{\rho}$ when finite element analysis is not available.

By substituting Eq. (21) into (26) and calculating the ratio of $J_{e}$ to $J_{e}^{\rho}$ it can be shown that

$J_{e}^{\rho}=J_{e}\left(1+\frac{1}{2 Y} \sqrt{\frac{\rho}{a}}\right)^{2}$

Eq. (28) can be used as a conservative method for calculating $J_{e}^{\rho}$.
The effect of notch radius on the limit load of various geometries is summarised in a comprehensive review by Miller [49]. Several expressions evaluate the influence of notch radius on limit load: the strongest (and therefore most conservative) effect is predicted for pure bending under plane strain conditions. Taking this as an example, using the Tresca yielding criterion,

$$
\begin{gathered}
\frac{4 M}{\sigma_{y} W^{2}(1-a / W)^{2}}=0.811+0.450\left(\frac{b}{b+\rho}\right) \text { for } \\
\\
0.64<\left(\frac{b}{b+\rho}\right)<1
\end{gathered}
$$

where $M$ is the moment/width and $b=W$ - $a$. By taking the ratio of the limit load for a notch to that of a crack, it can be shown that:

$P_{L}^{\rho}=P_{L}\left(0.643+0.357\left(\frac{b}{b+\rho}\right)\right)$ for $0.64<\left(\frac{b}{b+\rho}\right)<1$

A comparison of Eq. (30) with elastic-perfectly plastic finite element analysis of the notched $\mathrm{SE}(\mathrm{B})$ specimens shows good agreement, to within an accuracy of $2.7 \%$ for the range studied $\left(0.0004 \leq \rho / a_{0} \leq 0.32\right)$.

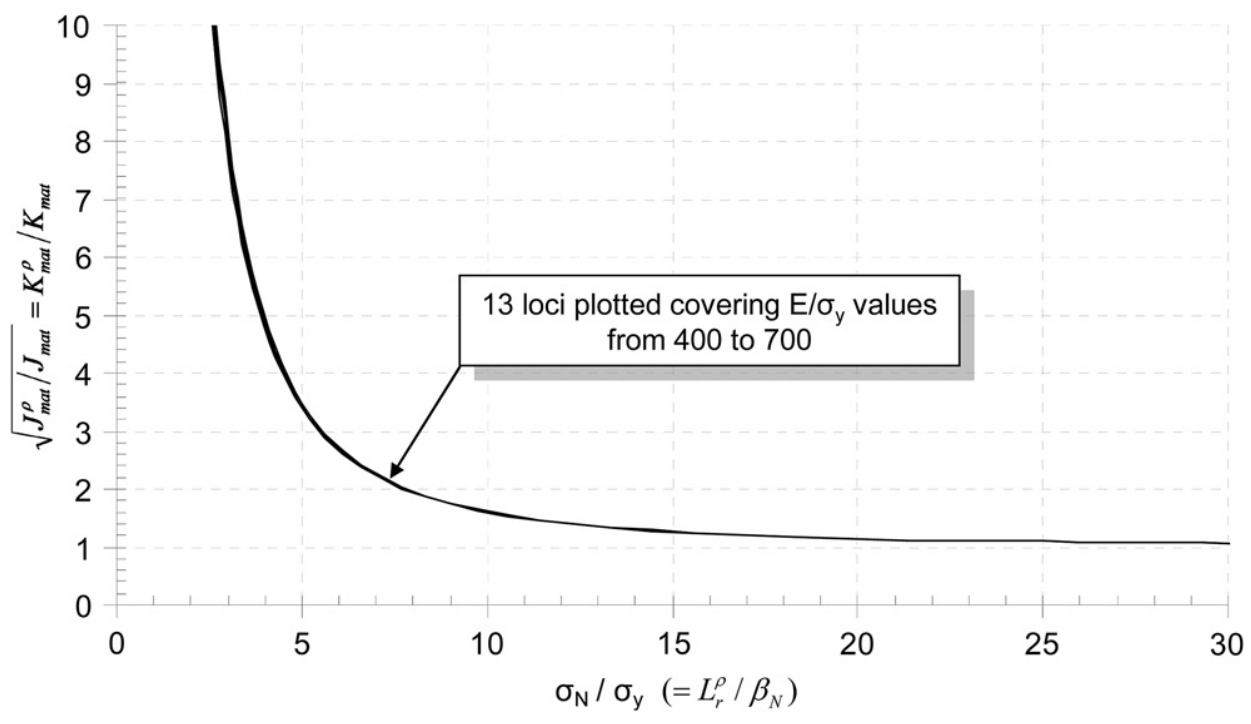

Fig. 12. Dependence of $K_{m a t}^{\rho} / K_{m a t}$ vs. $\sigma_{N} / \sigma_{y}$ as a function of $E / \sigma_{\mathrm{y}}$. 


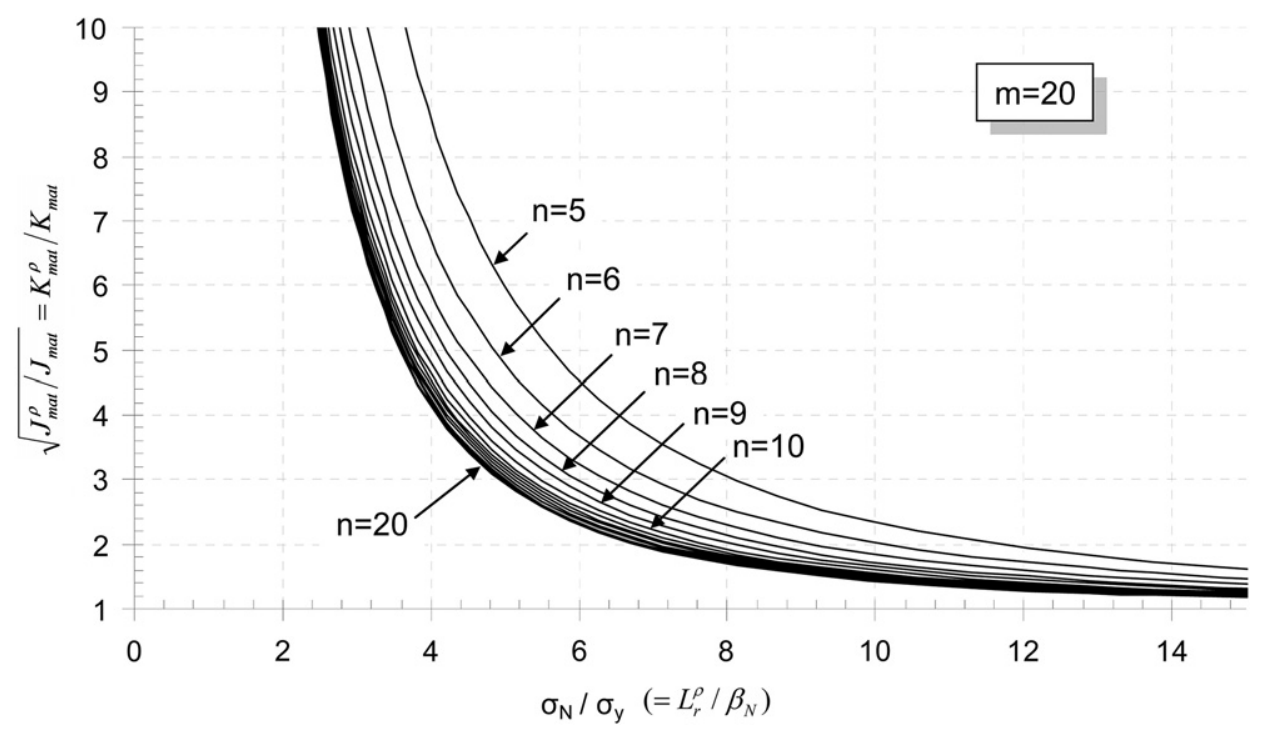

Fig. 13. Dependence of the $K_{m a t}^{\rho} / K_{m a t}$ vs. $\sigma_{N} / \sigma_{y}$ relationship on $n$ for $m=20$.

Other geometrical and loading configurations in reference [49] show a similar or weaker influence of $\rho$ on $P_{L}^{\rho}$ than that predicted by Eq. (30) It is therefore recommended that Eq. (30) is used as a conservative estimate.

\section{Results}

\subsection{Proposed FAD-based procedure for assessing non-sharp defects}

The proposed methodology for modifying the FAD for nonsharp defects comprises three steps, plus a preliminary "Step 0" analysis performed by assuming the non-sharp defect is a sharp crack. All parts of the procedure are applicable to both cleavage fracture and ductile tearing initiation with the exception of the look-up table in Step 3c which is only applicable to cleavage fracture. It has been outside the scope of the current work to develop a corresponding look-up table for ductile tearing initiation.

All the parameters required for the new assessment method can either be estimated using simple equations (using the "basic option") or they can be determined more accurately using finite element analysis (termed the "FE option").

\subsubsection{Step 0}

Assess the flaw by assuming it is a crack using the conventional FAD approach as detailed in R6 [1] or BS7910 [2]. Plot the assessment point on the FAD.

\subsubsection{Step 1}

Determine the notch root radius $\rho$. This can be done either by metallographic sectioning in cases where a sufficient quantity of flawed material is available, or in other cases by referencing distributions of previously measured $\rho$ values from the same type of flaw.

Determine the elastic notch driving force $J_{e}^{\rho}$. Using the "FE option", $J_{e}^{\rho}$ is determined using linear elastic FE analysis of the notched component at the load of interest where $J_{e}^{\rho}$ is equal to the path independent $J$-integral that completely encloses the notch root. Alternatively, the "basic option" can be used where $J_{e}^{\rho}$ is estimated conservatively from the elastic crack driving force $J_{e}\left(=K^{2}\left(1-\nu^{2}\right) / E\right)$ using Eq. (28).
Modify the $K_{r}$ coordinate of the assessment point according to

$K_{r}=\sqrt{\frac{J_{e}^{\rho}}{J_{\text {mat }}}}=\frac{K^{\rho}}{K_{\text {mat }}}$

This will result in a small increase in the $K_{r}$ coordinate of the assessment point.

\subsubsection{Step 2}

Determine the limit load for the notched structure $P_{L}^{\rho}$. Using the "FE option", elastic-perfectly plastic FE analysis of the notched component is performed where $P_{L}^{\rho}$ is equal to the maximum load obtained. Alternatively, using the "basic option", $P_{L}^{\rho}$ can be estimated from the limit load for a crack $P_{L}$ using Eq. (30). Modify the $L_{r}$ coordinate of the assessment point according to Eq. (9). This will result in a small increase in the $L_{r}$ coordinate of the assessment point.

\subsubsection{Step 3}

Obtain $J_{\text {mat }}^{\rho}$ from the following four sub-steps:

a. Calculate $\sigma_{\mathrm{N}}$ at the load of interest using either linear-elastic FE analysis of the notched component, or by using Eq. (21)

b. Calculate $\beta_{\mathrm{N}}$ using Eq. (12)

c. Obtain the material properties $\gamma$ and $l$. This can be done one of three ways:

- Perform material testing of U-notched SE(B) specimens having a range of notch tip radii and curve fitting to obtain $\gamma$ and $l$.

- For cleavage fracture, conservative estimates of $\gamma$ and $l$ can be obtained using Table 1 based on knowledge of $n$ and the Weibull modulus $m$.

- For cleavage fracture when $m$ is unknown but $n$ known, limited material testing of U-notched $\mathrm{SE}(\mathrm{B})$ specimens can be used in conjunction with Table 1

d. Calculate the effective notch toughness $K_{m a t}^{\rho}$ or $J_{m a t}^{\rho}$ using Eq. (11)

e. For cleavage fracture, if $K_{m a t}^{\rho}$ or $J_{m a t}^{\rho}$ calculated according to Eq. (11) is greater than the equivalent ductile tearing initiation value $K_{0.2}$ or $J_{0.2}, K_{\text {mat }}^{\rho}$ or $J_{\text {mat }}^{\rho}$ should be set equal to $K_{0.2}$ or $J_{0.2}$ respectively. This is necessary to avoid non-conservative assessments when $\rho$ is large enough to cause a transition in failure mechanism from cleavage to ductile tearing. 
The result can be shown on the FAD in two ways. The first option is modify the assessment point using Eq. (32):

$K_{r}^{\rho}=\sqrt{\frac{J_{e}^{\rho}}{J_{m a t}^{\rho}}}=\frac{K^{\rho}}{K_{m a t}^{\rho}}$

This will result in a reduction of $K_{r}$ moving the assessment point closer to the abscissa. In this approach, the failure assessment curve remains unchanged. However, since $K_{\text {mat }}^{\rho}$ is a function of $\sigma_{N}$ and hence applied load, the loading line becomes a non-linear function of $L_{r}$.

The alternative option is to leave the assessment point in its position calculated at the end of Step 2 and modify the failure locus according to Eq. (33)

$K_{r}^{\rho}=\left[1+\gamma\left(L_{r}^{\rho} / \beta_{N}\right)^{-l}\right] f\left(L_{r}^{\rho}\right)$

This results in a shift of the failure curve to higher values of $K_{r}$ by an amount which is dependent on the parameters $\gamma, l$ and $\sigma_{N}$.

\subsection{Look-up table}

Values of $\gamma$ and $\mathrm{l}$ as a function of $\mathrm{n}$ and $\mathrm{m}$ are given in Table 1 .

\section{Discussion}

\section{1. $J_{\text {mat }}^{\rho}$ As an effective toughness for non-sharp defects}

It is apparent from Fig. 4 that R6 Option 3 FAD loci for notched SE(B) specimens are broadly independent of $\rho$ when derived by referencing the notch $J$-integrals $J_{e}^{\rho}$ and $J^{\rho}$ (Eq. (8)). This observation is consistent with that of Wang et al. [16] who also observed that Option 3 failure assessment curves are virtually independent of $\rho$ for four different U-notched plate geometries subjected to tension. It logically follows, therefore, that in order to use existing FAD loci the $K_{r}$ coordinate of an assessment point should be calculated in a manner consistent with Eq. (8), i.e. Eq. (10).

In Section 2.2, it was shown that the notch $J$-integral could be estimated using existing methods derived for pre-cracked specimens. Fig. 3 shows that existing expressions in a range of fracture toughness testing standards provide reasonable estimates (to within $5 \%$ ) of the notch $J$-integral at failure, $J_{\text {mat }}^{\rho}$. For estimating notch $J$-integral values below the critical $J$ value, care should be taken to avoid partitioned expressions whose elastic components of $J$ result in underestimates of the total $J$-integral value (up to $20 \%$ for an $\mathrm{SE}(\mathrm{B})$ specimen with $\rho / a_{0}=0.32$ as shown in Fig. 3). In such cases, the non-partitioned expression in ESIS P2-92 [5] should be used (Eq. (3)) because this provides a reasonable estimate of the Jintegral (to within 5\%) at all levels of applied load.

\subsection{Physical meaning of J for a notch}

Although Rice's original definition of the J-integral was for a notch [24], the author is unaware of any physical notch growth mechanism by which a U-notch could propagate while retaining its original profile: when the mechanisms of cleavage fracture or ductile tearing act at the root of a blunt notch they effectively sharpen its root profile, so the physical meaning of $J$ for a notch is unclear.

Table 1

Lookup table for $\gamma$ and $l$.

\begin{tabular}{|c|c|c|c|c|c|c|c|c|c|c|}
\hline & \multicolumn{2}{|l|}{$m=5$} & \multicolumn{2}{|l|}{$m=7.5$} & \multicolumn{2}{|c|}{$m=10$} & \multicolumn{2}{|c|}{$m=12.5$} & \multicolumn{2}{|c|}{$m=15$} \\
\hline & $\gamma$ & $l$ & $\gamma$ & l & $\gamma$ & $l$ & $\gamma$ & $l$ & $\gamma$ & $l$ \\
\hline$n=5$ & 8.62 & 2.61 & 7.34 & 1.94 & 11.54 & 1.76 & 19.3 & 1.71 & 33.5 & 1.74 \\
\hline$n=6$ & 6.38 & 2.52 & 6.48 & 1.96 & 9.82 & 1.78 & 16.1 & 1.73 & 26.9 & 1.74 \\
\hline$n=7$ & 5.59 & 2.52 & 6.27 & 2.00 & 9.77 & 1.83 & 15.7 & 1.78 & 25.5 & 1.79 \\
\hline$n=8$ & 5.08 & 2.52 & 6.14 & 2.03 & 9.76 & 1.87 & 15.8 & 1.83 & 25.2 & 1.84 \\
\hline$n=9$ & 4.73 & 2.53 & 6.02 & 2.04 & 9.75 & 1.91 & 15.8 & 1.87 & 25.3 & 1.89 \\
\hline$n=10$ & 4.37 & 2.52 & 5.91 & 2.05 & 9.77 & 1.93 & 16.0 & 1.90 & 25.5 & 1.92 \\
\hline$n=11$ & 3.94 & 2.50 & 5.81 & 2.06 & 9.80 & 1.95 & 16.1 & 1.93 & 25.7 & 1.95 \\
\hline$n=12$ & 3.65 & 2.48 & 5.72 & 2.07 & 9.85 & 1.97 & 16.1 & 1.95 & 25.9 & 1.98 \\
\hline$n=13$ & 3.37 & 2.47 & 5.66 & 2.07 & 9.87 & 1.98 & 16.2 & 1.97 & 26.0 & 1.99 \\
\hline$n=14$ & 2.99 & 2.44 & 5.57 & 2.07 & 9.88 & 1.99 & 16.2 & 1.98 & 26.1 & 2.01 \\
\hline$n=15$ & 2.74 & 2.42 & 5.48 & 2.08 & 9.87 & 2.00 & 16.3 & 1.99 & 26.3 & 2.02 \\
\hline$n=16$ & 2.54 & 2.40 & 5.42 & 2.08 & 9.87 & 2.00 & 16.4 & 2.00 & 26.4 & 2.03 \\
\hline$n=17$ & 2.32 & 2.37 & 5.37 & 2.07 & 9.85 & 2.00 & 16.4 & 2.01 & 26.5 & 2.04 \\
\hline$n=18$ & 2.18 & 2.34 & 5.32 & 2.07 & 9.84 & 2.01 & 16.4 & 2.01 & 26.6 & 2.05 \\
\hline$n=19$ & 2.07 & 2.32 & 5.26 & 2.07 & 9.81 & 2.00 & 16.4 & 2.01 & 26.6 & 2.05 \\
\hline \multirow[t]{3}{*}{$n=20$} & 1.96 & 2.30 & 5.20 & 2.06 & 9.74 & 2.00 & 16.3 & 2.01 & 26.3 & 2.05 \\
\hline & \multicolumn{2}{|c|}{$m=17.5$} & \multicolumn{2}{|l|}{$m=20$} & \multicolumn{2}{|c|}{$m=22.5$} & \multicolumn{2}{|c|}{$m=25$} & \multicolumn{2}{|c|}{$m=27.5$} \\
\hline & $\gamma$ & $l$ & $\gamma$ & $l$ & $\gamma$ & $l$ & $\gamma$ & $l$ & $\gamma$ & $l$ \\
\hline$n=5$ & 59.0 & 1.80 & 106 & 1.90 & 189 & 2.01 & 343 & 2.14 & 624 & 2.28 \\
\hline$n=6$ & 45.5 & 1.80 & 78.1 & 1.88 & 134 & 1.99 & 234 & 2.10 & 406 & 2.23 \\
\hline$n=7$ & 42.5 & 1.85 & 70.1 & 1.92 & 117 & 2.02 & 197 & 2.13 & 331 & 2.24 \\
\hline$n=8$ & 41.1 & 1.89 & 67.6 & 1.97 & 110 & 2.05 & 183 & 2.17 & 296 & 2.27 \\
\hline$n=9$ & 40.7 & 1.93 & 65.9 & 2.01 & 106 & 2.09 & 172 & 2.19 & 280 & 2.29 \\
\hline$n=10$ & 40.8 & 1.97 & 64.3 & 2.04 & 104 & 2.13 & 168 & 2.23 & 270 & 2.32 \\
\hline$n=11$ & 41.0 & 2.00 & 64.3 & 2.07 & 104 & 2.16 & 167 & 2.26 & 267 & 2.36 \\
\hline$n=12$ & 41.2 & 2.03 & 64.8 & 2.10 & 104 & 2.19 & 167 & 2.29 & 265 & 2.40 \\
\hline$n=13$ & 41.5 & 2.05 & 65.4 & 2.12 & 105 & 2.21 & 167 & 2.32 & 264 & 2.43 \\
\hline$n=14$ & 41.7 & 2.07 & 65.8 & 2.14 & 105 & 2.23 & 166 & 2.34 & 263 & 2.44 \\
\hline$n=15$ & 41.8 & 2.08 & 66.4 & 2.16 & 105 & 2.25 & 166 & 2.35 & 263 & 2.46 \\
\hline$n=16$ & 42.1 & 2.10 & 66.6 & 2.17 & 105 & 2.26 & 166 & 2.37 & 262 & 2.47 \\
\hline$n=17$ & 42.4 & 2.11 & 66.6 & 2.18 & 105 & 2.27 & 165 & 2.38 & 262 & 2.48 \\
\hline$n=18$ & 42.6 & 2.12 & 66.7 & 2.19 & 104 & 2.28 & 166 & 2.38 & 261 & 2.49 \\
\hline$n=19$ & 42.7 & 2.12 & 66.6 & 2.19 & 104 & 2.28 & 165 & 2.39 & 261 & 2.50 \\
\hline$n=20$ & 42.7 & 2.12 & 66.3 & 2.20 & 102 & 2.28 & 164 & 2.39 & 260 & 2.50 \\
\hline
\end{tabular}



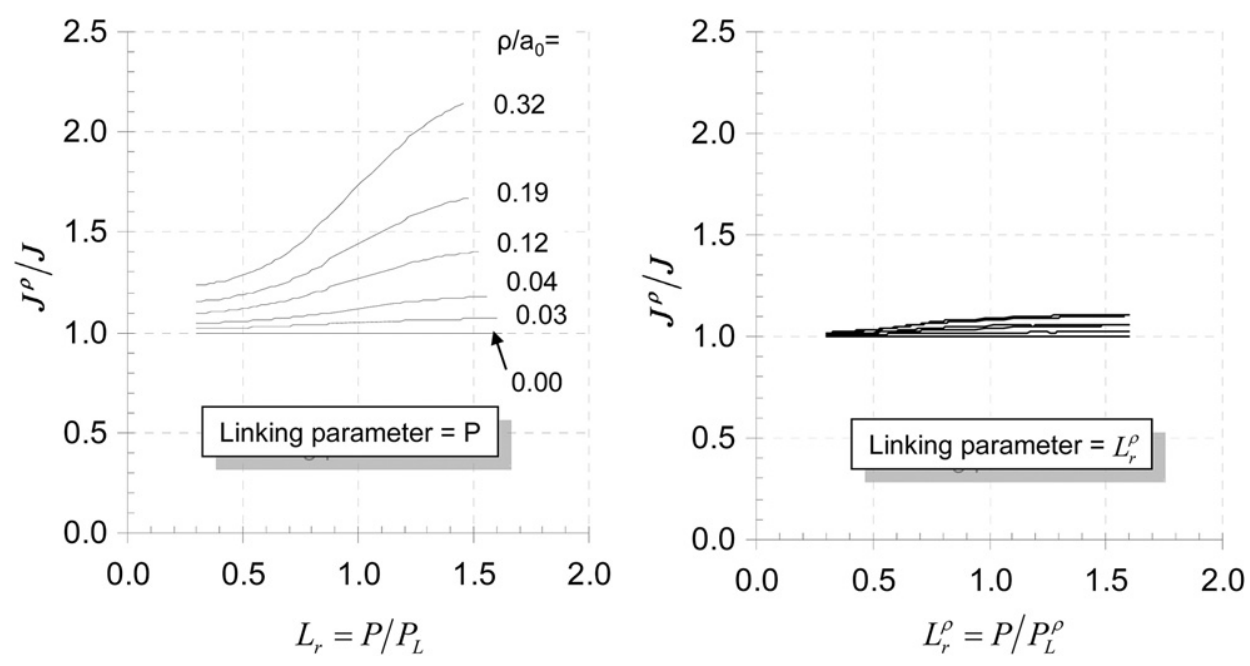

Fig. 14. Comparison of different linking parameters for defining an 'equivalent' cracked specimen.

It is possible to interpret the value of $J$ by relating the notched specimen to an 'equivalent' cracked specimen for which $J$ does have a clear physical meaning. A variety of different linking parameters can be used to define what is meant by an 'equivalent' cracked structure. The example of load is shown on the left hand side of Fig. 14: the ratio of $J^{\rho}$ to the value of $J$ in an equivalent cracked structure subject to the same load is plotted as function of $L_{r}$. This however does not result in a good correlation: at high $L_{r}, J^{\rho}$ for $\rho / a_{0}=0.32$ is over twice the equivalent sharp crack value of $J$.

The right hand side of Fig. 14 shows the ratio of $J^{\rho} / J$ but defined at the same $L_{r}^{\rho}$ rather than load, i.e. normalising the load for each notched specimen to the relevant limit load $P_{L}^{\rho}$ for that specimen. This results in a much closer correlation between $J^{\rho}$ and $J$. For acute notches with $0 \leq \rho / a_{0} \leq 0.12, J^{\rho}$ is within $5 \%$ of the equivalent sharp crack value $J$. The relationship is less close for blunter notches: for $0.12<\rho / a_{0} \leq 0.32, J^{\rho}$ is between 5 and $11 \%$ greater than $J$ defined at the same $L_{r}^{\rho}$. This relationship suggests that, for acute notches, $J^{\rho}$ can be interpreted as the value of $J$ that an equivalent cracked structure would have if subjected to the same $L_{r}^{\rho}$.

So, although the physical meaning of $J^{\rho}$ is unclear specifically for a notched specimen, $J^{\rho}$ can be interpreted as the value of $J$ an equivalent cracked specimen would have at the same value of $L_{r}^{\rho}$ (for acute notches with $0 \leq \rho / a_{0} \leq 0.12$ where values are within $5 \%$ of each other). Furthermore:

- Option 3 failure assessment curves are broadly independent of $\rho$ because the ratio $J_{e}^{\rho} / J^{\rho}$ is independent of $\rho$ when defined at the same $L_{r}^{\rho}$

- Existing non-partitioned experimental expressions for $J$ can be applied to notched $\mathrm{SE}(\mathrm{B})$ specimens to provide reasonably accurate estimations of $J^{\rho}$ (to within 5\%).

- $J^{\rho}$ is proportional to the physical distance between the notch flanks at a specific position

It is therefore reasonable to use $J^{\rho}$ as a measure of the notch driving force.

\section{3. $\rho / a_{0}$ limits}

An important issue to consider is the range of $\rho / a_{0}$ values over which the new assessment methodology will remain accurate. Whereas the new assessment procedure is most accurate for the limiting case of a sharp crack where $\rho / a_{0} \rightarrow 0$, it will become less accurate as $\rho / a_{0}$ increases. The range of valid $\rho / a_{0}$ values of a given level of accuracy will also depend on whether the "basic option" or "FE option" is used.

An important basis of the new methodology is the observation that the failure assessment lines are almost independent of notch root radius, first observed by Wang et al. [16] for loading in tension for $0 \leq \rho / a_{0} \leq 0.20$ and confirmed in the current work for loading in bending for $0 \leq \rho / a_{0} \leq 0.32$. Although the limits to $\rho / a_{0}$ have not been rigorously established in the current work, it is recommended that the new assessment procedure is not used to assess notched structures with $\rho / a_{0}$ values outside these ranges, unless the failure assessment lines can be shown to be broadly independent of $\rho$. If this can be demonstrated, additional caution should be exercised if using the "basic option" outside the $\rho / a_{0}$ range used in the current work: although the accuracy of the equations developed in Section 2.6 has been quantified for $0 \leq \rho / a_{0} \leq 0.32$, they are likely to become less accurate for $\rho / a_{0}>0.32$.

\section{Conclusions}

A new engineering assessment method has been proposed for predicting failure in structures that contain non-sharp, slender (i.e. $\rho / a_{0} \leq 0.32$ ) defects with parallel notch flanks and smoothly turning tips. A modification of the Failure Assessment Diagram (FAD) for non-sharp defects has been proposed which can be applied irrespective of failure mechanism. The main benefit arises from the increase in effective toughness with increasing notch radius. Although this benefit appears whether the initiating failure mechanism is cleavage or ductile tearing, this paper focuses on cleavage fracture. In the new assessment procedure, the notch driving force is described by the notch J-integral $J^{\rho}$, the notch tip loading severity by the elastic notch tip opening stress $\sigma_{\mathrm{N}}$ and the notch geometry by a load-independent parameter $\beta_{\mathrm{N}}$. The sensitivity of the material toughness to the notch effect is defined by the non-dimensional material parameters $\gamma$ and $l$.

The following conclusions can be drawn from the work presented in this Part I paper:

1. The value of $J^{\rho}$ in $U$-notched $\mathrm{SE}(\mathrm{B})$ specimens can be estimated using existing expressions derived for pre-cracked specimens. Non-partitioned expressions provide the most accurate estimation of $J^{\rho}$.

2. R6 Option 3 failure assessment curves defined according to $f_{3}\left(L_{r}^{\rho}\right)=\sqrt{J_{e}^{\rho} / J^{\rho}}$ are broadly independent of $\rho$ allowing existing failure loci to be used in the notch-modified FAD procedure. 
3. The elastic notch tip opening stress $\sigma_{N}$ is used as a parameter to describe the notch tip loading severity for three reasons. Firstly, $\sigma_{N}$ is proportional to load with a gradient $1 / \beta_{N}$ that is a unique function of the notch geometry. Secondly, $\sigma_{N}$ can be accurately estimated using existing expressions in the literature. Finally, for any value of $\sigma_{N}$, the cleavage toughness benefit due to the notch effect is independent of load, Weibull stress and therefore failure probability.

4. The non-dimensional material parameters $\gamma$ and $l$ define the sensitivity of the material toughness to the notch effect. These parameters can either be measured by testing notched specimens of the same thickness as the structure, or for cleavage fracture they can be obtained using look-up tables generated using the Weibull stress toughness scaling model.

5. The other parameters in the procedure can either be conservatively estimated using simple equations or they can be determined more accurately using finite element analysis.

A series of worked examples using a large dataset of notched $\mathrm{SE}(\mathrm{B})$ specimens are presented in the companion Part II paper [31]. The Part II paper also considers the relationship between the new FAD modified for non-sharp defects and the constraint-modified FAD approach detailed in R6. A combined notch and constraintmodified FAD is presented and compared with test data on a series of shallow, blunt notch $\mathrm{SE}(\mathrm{B})$ specimens.

\section{Acknowledgements}

The authors wish to thank Tata Steel for funding the work, and to acknowledge the help of Dr. Martin Goldthorpe of the Materials Performance Centre, The University of Manchester, for useful discussions relating to the modelling aspects of the work. The authors also wish to thank Prof. Bob Ainsworth of The University of Manchester for invaluable discussions on the incorporation of the notch effect into the Failure Assessment Diagram. The helpful assistance of Dr. Peter Budden and Dr. Yuebo Lei from EDF Energy, Mr. David Beardsmore from Serco Technical and Assurance Services, and Prof. John Hancock from Glasgow University is also gratefully acknowledged.

\section{Appendix A}

This appendix derives scaling functions such that the $\sigma_{\mathrm{w}}$ vs. $J^{\rho}$ relationship obtained from one boundary layer analysis with notch root radius $\rho$ can be scaled to any other notch root radius $\rho^{\prime}$ while keeping the notch front length constant.

The standard Weibull stress equation, assuming all inputs are in $\mathrm{SI}(\mathrm{mm})$ units, is:
If the results of a finite element model were interpreted by assuming that all inputs had been specified using the $\mathrm{SI}(\mathrm{m})$ unit system as an alternative unit system to $\mathrm{SI}(\mathrm{mm})$, all distances would be 1000 times greater than in the original model, i.e. a distance multiplication factor $F=1000$. In this case, $V_{0(\mathrm{SC})}, \sigma_{1(\mathrm{SC})}$ and $\sigma_{\mathrm{W}(\mathrm{SC})}$ would scale in the following way:

- $\mathrm{V}_{0}$ must be kept constant for the Weibull parameters to be comparable. Therefore, the value of $V_{0(\mathrm{SC})}$ must be kept equal to $V_{0}$ (i.e. $1 \mathrm{~mm}^{3}$ ), but expressed in units of $\mathrm{m}^{3}$. For a constant notch front length:

$V_{0(S C)}=V_{0} F^{-2}$

- Using $\mathrm{SI}(\mathrm{m})$ units, the stress-strain curve specified in ABAQUS will be in units of Pa (compared to MPa when using $\mathrm{SI}(\mathrm{mm})$ units): the material modelled using the alternative unit system therefore has less strength that the actual material modelled using $\mathrm{SI}(\mathrm{mm})$ units $\left(\sigma_{\mathrm{y}}=430 \mathrm{~Pa}\right.$ rather than $\left.\sigma_{\mathrm{y}}=430 \mathrm{MPa}\right)$. In order to scale the "weak" material up to the actual material, all stresses must be multiplied by $\mathrm{F}^{2}$, i.e.:

$\sigma_{1(S C)}=\sigma_{1} F^{2}$

- Using $\mathrm{SI}(\mathrm{m})$ units, the calculated Weibull stress $\sigma_{\mathrm{W}(\mathrm{SC})}$ according to Eq. (A2) will be in be in units of Pa by default. To change the units to MPa, thereby allowing direct comparison with Eq. (A1), $\sigma_{\mathrm{w}(\mathrm{SC})}$ must be divided by $\mathrm{F}^{2}$, i.e.:

$\sigma_{w(S C, M P a)}=\sigma_{w(S C)} F^{-2}$

Substituting Eqs. (A3), (A4) and (A5) into Eq. (A2) gives:

$\sigma_{w(S C, M P a)}=F^{-2}\left[\frac{F^{2}}{V_{0}} \int_{V}\left(\sigma_{1} F^{2}\right)^{m} \mathrm{~d} V\right]^{1 / m}$

By raising both sides to the power $m$ the equation becomes

$\sigma_{w(S C, M P a)}^{m}=F^{-2 m}\left[\frac{F^{2} F^{2 m}}{V_{0}} \int_{V} \sigma_{1}^{m} \mathrm{~d} V\right]$

The terms $F^{2 \mathrm{~m}}$ and $F^{-2 \mathrm{~m}}$ cancel each other out, leaving the following expression:

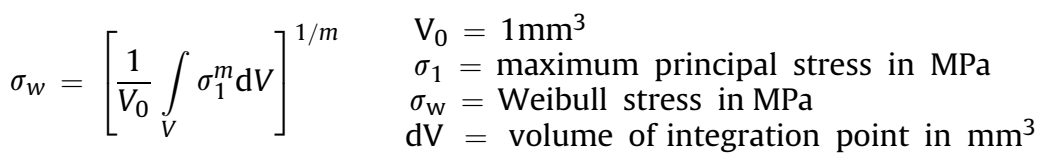

If an alternative unit system were to be used, the above equation would require modification as follows:

$\sigma_{w(S C)}=\left[\frac{1}{V_{0(S C)}} \int_{V} \sigma_{1(S C)}^{m} \mathrm{~d} V\right]^{1 / m} \quad \begin{aligned} \mathrm{V}_{0(\mathrm{SC})} & =\text { scaled equivalent of } \mathrm{V}_{0} \text { in alternative units } \\ \sigma_{1(\mathrm{SC})} & =\text { scaled equivalent of } \sigma_{1} \text { in alternative units } \\ \sigma_{\mathrm{w}(\mathrm{SC})} & =\text { scaled equivalent of } \sigma_{\mathrm{w}} \text { in alternative units }\end{aligned}$ 
$\sigma_{w(S C, M P a)}^{m}=\frac{F^{2}}{V_{0}} \int_{V} \sigma_{1}^{m} \mathrm{~d} V$

Diving Eq. (A6) by Eq. (A1) leads to

$\frac{\sigma_{w(S C, M P a)}^{m}}{\sigma_{w}^{m}}=F^{2}$

i.e. $\frac{\sigma_{w(S C, M P a)}}{\sigma_{w}}=F^{2 / m}$

\section{References}

[1] R6: assessment of the integrity of structures containing defects, Revision 4. British Energy Generation Limited; 2005.

[2] BSI. BS 7910: Guide on methods for assessing the acceptability of flaws in metallic structures. British Standards Institute; 2005.

[3] BSI. BS 7448-1: fracture mechanics toughness tests - Part 1: method for determination of KIC, critical CTOD and critical J values of metallic materials. British Standards Institute; 1999.

[4] ASTM. ASTM E1921-08: standard test method for determination of reference temperature, To, for ferritic steels in the transition range. ASTM; 2008.

[5] ESIS. ESIS P2-92: ESIS procedure for determining the fracture behaviour of materials; 1992

[6] Milne I, Chell GG, Worthington PJ. The mechanisms of fracture in bluntnotched specimens of a low-alloy steel and the effect on failure assessment. Materials Science and Engineering 1979;40:145-57.

[7] Gomez FJ, Elices M. A fracture criterion for blunted V-notched samples. International Journal of Fracture 2004;127:239-64.

[8] Spink GM, Worthington PJ, Heald PT. The effect of notch acuity on fracture toughness testing. Materials Science and Engineering 1973;11:113-7.

[9] Livieri P. Use of J-integral to predict static failures in sharp V-notches and rounded U-notches. Engineering Fracture Mechanics 2008;75(7):1779-93.

[10] Srinivas M, Kamat SV, Ramo Rao R. Effect of notch root radius on ductile fracture toughness of Armco Iron. International Journal of Fracture 1992;58.

[11] Yoda M. The effect of notch root radius on the J-integral fracture toughness under modes I, II and III loadings. Engineering Fracture Mechanics 1987;26: 425-31.

[12] Faucher B, Tyson WR, Hong Y, Boutin J. Dependence of ductile fracture toughness of a Weld Metal on notch root radius and Inclusion Content. International Journal of Fracture 1990;46:173-84.

[13] Kamat SV, Srinivas M. Investigation of blunting line and evaluation of fracture toughness under mixed mode I/III loading in commercially pure titanium. Materials Science and Technology 2003;19(1):62-6.

[14] Horn AJ, Sherry AH. Prediction of cleavage fracture from non-sharp defects using the Weibull stress based toughness scaling model. International Journal of Pressure Vessels and Piping 2010;87:670-80.

[15] Taylor D, Cornetti P, Pugno N. The fracture mechanics of finite crack extension. Engineering Fracture Mechanics 2005;72(7):1021-38.

[16] Wang WQ Li AJ, Li PN, Ju DY. Engineering approach for notch elastic-plastic fracture analysis. International Journal of Pressure Vessels and Piping 1994; 60:1-16.

[17] Smith E. Fracture initiation at the root of a blunt flaw: description in terms of $\mathrm{Kr}-\mathrm{Lr}$ failure assessment curves. International Journal of Pressure Vessels and Piping 1999;76(11):799-800.

[18] Matvienko YG. Local fracture criterion to describe failure assessment diagrams for a body with a crack/notch. International Journal of Fracture 2003;124: 107-12.

[19] Cicero S, Gutierrez-Solana F, Horn AJ. Experimental analysis of differences in mechanical behaviour of cracked and notched specimens in a ferritic-pearlitic steel: Considerations about the notch effect on structural integrity. Engineering Failure Analysis 2009;16:2450-66.

[20] Cicero S, Gutiérrez-Solana F, Álvarez JA. Structural integrity assessment of components subjected to low constraint conditions. Engineering Fracture Mechanics 2008;75(10):3038-59.

[21] Kocak M, Webster S, Janosch JJ, Ainsworth RA, Koers R. FITNET Fitness-forService procedure - Version MK8. GKSS; 2006.
[22] Nui LS, Chehimi C, Pluvinage G. Stress field near a large blunted tip V-notch and application of the concept of the critical notch stress intensity factor (NSIF) to the fracture toughness of very brittle materials. Engineering Fracture Mechanics 1994;49(3):325-35.

[23] Susmel L, Taylor D. On the use of the Theory of Critical Distances to predict static failures in ductile metallic materials containing different geometrical features. Engineering Fracture Mechanics 2008;75(15):4410-21.

[24] Rice JR. A path independent integral and the approximate analysis of strain concentration by notches and cracks. Journal of Applied Mechanics 1968;35: 379-86.

[25] ASTM. ASTM E1820-08a: standard test method for measurement of fracture toughness. ASTM; 2008

[26] Matvienko YG, Morozov EM. Calculation of the energy J-integral for bodies with notches and cracks. International Journal of Fracture 2004;125(3-4): 249-61.

[27] Chen YH, Lu TJ. On the path dependence of the J-integral in notch problems. International Journal of Solids and Structures 2004;41(3-4):607-18.

[28] Ranaweera MP, Leckie FA. J Integrals for some crack and notch geometries. International Journal of Fracture 1982;18(1):3-18

[29] Schindler HJ. Determination of fracture mechanics material properties utilizing notched test specimens. ICM; 1991.

[30] Roberti R, Silva G, Firrao D, de Benedetti B. Influence of notch root radius on ductile rupture fracture toughness evaluation with Charpy-V type specimens. International Journal of Fatigue 1981;3(3):133-41.

[31] Horn AJ, Sherry AH. An engineering assessment methodology for non-sharp defects in steel structures - Part II: Procedure validation and constraint analysis. International Journal of Pressure Vessels and Piping 2012;89: 151-61.

[32] Gao X, Dodds RHJ. An engineering approach to assess constraint effects on cleavage fracture toughness. Engineering Fracture Mechanics 2001;68:263-83.

[33] ABAQUS/Standard v6.6-1. Hibbitt. Karlsson and Sorenson Inc; 2006.

[34] Nevalainen M, Dodds Jr RH. Numerical investigation of 3-D constraint effects on brittle fracture in $S E(B)$ and $C(T)$ specimens. International Journal of Fracture 1995;74(2):131-61.

[35] Brocks W, Scheider I. Reliable J values: numerical aspects of the path dependence of the J integral in Incremental Plasticity. 2003/22. GKSS; 2003.

[36] ISO. ISO 15653: metallic Materials - Method of test for the determination of quasistatic fracture toughness of welds. International Standards Organisation; 2009.

[37] Beremin FM. A Local criterion for cleavage fracture of a Nuclear Pressure Vessel steel. Metallurgical Transactions A 1983;14A:2277-87.

[38] Gao X, Ruggieri C, Dodds Jr RH. Calibration of Weibull stress parameters using fracture toughness data. International Journal of Fracture 1998;92(2): $175-200$.

[39] Williams ML. Stress singularities resulting from various boundary conditions in angular corners of plates in extension. Journal of Applied Mechanics 1952; 19:526-8.

[40] Sherry AH, Wilkes MA, Beardsmore DW, Lidbury DPG. Material constraint parameters for the assessment of shallow defects in structural components Part I: parameter solutions. Engineering Fracture Mechanics 2005;72: 2373-95.

[41] Bannister A. Yield stress to tensile stress ratio: results of experimental programme. British Steel Technical, Swinden Technology Centre; 1999. Report No. BRPR-CT95-0024

[42] Sherry AH, Hooton DG, Beardsmore DW, Lidbury DPG. Constraint-Based Assessments: VOCALIST validation of constraint based assessment methodology in structural integrity; December 2003.

[43] Shin CS. A discussion on various estimations of elastic stress distributions and stress concentration factors for sharp edge notches. International Journal of Fatigue 1986;8(4):235-7.

[44] Creager M, Paris PC. Elastic field equations for blunt cracks with reference to stress corrosion cracking. International Journal of Fracture Mechanics 1967;3: 247-52.

[45] Murikami Y. Stress intensity factors Handbook. Permagon Books Ltd; 1987.

[46] Berto F, Lazzarin P, Matvienko YG. J-integral evaluation for U- and V-blunt notches under Mode I loading and materials obeying a power hardening law. International Journal of Fracture 2007;146(1-2):33-51.

[47] Szanto M, Read DT. J-integral applied to stress-strain concentrations. Engineering Fracture Mechanics 1992:43(3):401-15.

[48] Molski K, Glinka G. A method of elastic-plastic stress and strain calculation at a notch root. Materials Science and Engineering 1981;50(1):93-100.

[49] Miller AG. Review of limit loads of structures containing defects. International Journal of Pressure Vessels and Piping 1988;32:197-327. 